\title{
REFLECTION OF PROPAGATING SLOW MAGNETO-ACOUSTIC WAVES IN HOT CORONAL LOOPS: MULTI-INSTRUMENT OBSERVATIONS AND NUMERICAL MODELING
}

\author{
Sudip Mandal ${ }^{1}$, Ding Yuan $^{2,3}$, Xia Fang $^{2}$, Dipankar Banerjee ${ }^{1,4}$, Vaibhav Pant ${ }^{1}$, and Tom Van Doorsselaere ${ }^{2}$ \\ ${ }^{1}$ Indian Institute of Astrophysics, Koramangala, Bangalore 560034, India; sudip@iiap.res.in \\ ${ }^{2}$ Centre for mathematical Plasma Astrophysics, Department of Mathematics, KU Leuven, Celestijnenlaan 200B, bus 2400, 3001, \\ Leuven, Belgium; xia.fang@wis.kuleuven.be \\ ${ }^{3}$ Jeremiah Horrocks Institute, University of Central Lancashire, Preston PR1 2HE, UK \\ ${ }^{4}$ Center of Excellence in Space Sciences India, IISER Kolkata, Mohanpur 741246, West Bengal, India \\ Received 2016 January 19; revised 2016 May 18; accepted 2016 May 20; published 2016 September 2
}

\begin{abstract}
Slow MHD waves are important tools for understanding coronal structures and dynamics. In this paper, we report a number of observations from the X-Ray Telescope (XRT) on board HINODE and Solar Dynamic Observatory/ Atmospheric Imaging Assembly (AIA) of reflecting longitudinal waves in hot coronal loops. To our knowledge, this is the first report of this kind as seen from the XRT and simultaneously with the AIA. The wave appears after a micro-flare occurs at one of the footpoints. We estimate the density and temperature of the loop plasma by performing differential emission measure (DEM) analysis on the AIA image sequence. The estimated speed of propagation is comparable to or lower than the local sound speed, suggesting it to be a propagating slow wave. The intensity perturbation amplitude, in every case, falls very rapidly as the perturbation moves along the loop and eventually vanishes after one or more reflections. To check the consistency of such reflection signatures with the obtained loop parameters, we perform a 2.5D MHD simulation, which uses the parameters obtained from our observation as inputs, and perform forward modeling to synthesize AIA $94 \AA$ images. Analyzing the synthesized images, we obtain the same properties of the observables as for the real observation. From the analysis we conclude that a footpoint heating can generate a slow wave which then reflects back and forth in the coronal loop before fading. Our analysis of the simulated data shows that the main agent for this damping is anisotropic thermal conduction.
\end{abstract}

Key words: magnetohydrodynamics (MHD) - Sun: corona - Sun: flares - Sun: magnetic fields - Sun: oscillations - Sun: UV radiation

Supporting material: animations

\section{INTRODUCTION}

MHD waves play an important role in understanding solar structures and the coronal heating process (Roberts et al. 1984; Nakariakov \& Verwichte 2005; Banerjee et al. 2007; De Moortel \& Nakariakov 2012). Extreme ultra-violet (EUV) imaging analysis provides access to the loop diagnostics, i.e loop density, temperature, and flows, including estimation of magnetic field (Roberts et al. 1984; Aschwanden et al. 1999; Nakariakov et al. 1999; Nakariakov \& Ofman 2001; Verwichte et al. 2013). Slow MHD waves in the solar corona were first observed by Ofman et al. (1997), DeForest \& Gurman (1998) and Berghmans \& Clette (1999) as quasi-periodic propagating disturbances (PDs) channeling through coronal structures. These PDs have an apparent speed close to the sound speed of that medium, leading to their explanation as propagating slow waves (Marsh \& Walsh 2009; Marsh et al. 2009; Kiddie et al. 2012). Using simultaneous imaging and spectroscopic data, recent analysis, including "red-blue (R-B)" asymmetry, shows that the upflow scenario could also explain the observed PDs (De Pontieu et al. 2009; Tian et al. 2011; Mandal et al. 2015). Recently it has been shown by Verwichte et al. (2010) that slow waves can also naturally explain the behavior of the $\mathrm{R}-\mathrm{B}$ parameter.

Standing slow waves have also been studied very closely by many authors. Wang et al. (2002, 2003a, 2003b) observed damped Doppler shift oscillations in hot coronal lines with SOHO/SUMER. They interpreted these oscillatory Doppler shifts of high temperature ( $T>6 \mathrm{MK}$ ) Fe XIX and Fe XXI lines as standing slow waves generated by an impulsive trigger at one of the loop footpoints. Wang et al. (2005) analyzed 54 Doppler shift oscillations in 27 flare-like events from SOHO/ SUMER and interpreted them as being caused by standing slow waves because they exhibit a quarter period phase shift between the intensity and velocity oscillations. Mariska (2005) reported Doppler shift oscillations in S XV and Ca XIX lines observed with BCS/Yohkoh where they found oscillations with a period of few minutes (Mariska 2006) (from HINODE/ EIS Mariska et al. 2008). Using forward modeling, Yuan et al. (2015) synthesized Solar Dynamic Observatory (SDO)/Atmospheric Imaging Assembly (AIA) and $\mathrm{SOHO} / \mathrm{SUMER}$ emissions to study standing slow wave modes in a hot flaring loop. Apart from recovering the quarter period phase shift between intensity and Doppler velocity, these authors also found asymmetric emission intensity during the positive and negative temperature perturbation phase.

Selwa et al. (2005) generated slow waves numerically by applying temperature perturbation pulses at different positions in the loop and observed that the generated mode depends upon the location of the pulses. The study of various aspects of slow wave generation have been extensively carried out using 2D and 3D MHD models (Selwa et al. 2007; Ofman \& Selwa 2009; Selwa \& Ofman 2009; Ofman et al. 2012). Using a 3D MHD model Ofman et al. (2012) concluded that the impulsive injection of energy at the active region loop footpoint can excite slow and fast waves simultaneously along with the observed outflows. Damping of the Doppler oscillations 
Table 1

Details of The XRT ("Be-thin") Observations

\begin{tabular}{|c|c|c|c|c|c|}
\hline Date & Time (UT) & Active Region & FOV & Cadence (s) & $\overline{\text { Pixel scale }}$ \\
\hline 2015 Dec 10 & $04: 30-05: 16$ & AR 12465 & $2106^{\prime \prime} \times 2106^{\prime \prime}$ & 121 & $4.1^{\prime \prime}$ \\
\hline 2013 Jan 22 & $08: 30-09: 29$ & AR 11654 & $394^{\prime \prime} \times 394^{\prime \prime}$ & 61 & $1.03^{\prime \prime}$ \\
\hline 2013 Jan 27 & 20:09-20:59 & AR 11661 & $394^{\prime \prime} \times 394^{\prime \prime}$ & 61 & $1.03^{\prime \prime}$ \\
\hline
\end{tabular}

(observed with SUMER) has also been studied thoroughly by Ofman \& Wang (2002). Using a 1D MHD code simulation, these authors concluded that thermal conduction plays a significant role in damping these waves, rather than compressive viscosity. Damping of the waves has been further studied by De Moortel \& Hood $(2003,2004)$ and De Moortel et al. (2004) where they included gravitational stratification, field line divergence and mode coupling apart from thermal conduction and viscosity.

Reflection of slow MHD waves propagating through coronal loops has been reported recently by Kumar et al. (2013) using high-resolution imaging data from the $S D O /$ AIA high temperature 131 and $94 \AA$ channels. A slow wave generated at one of the footpoints was reflected back and forth a couple of times from the loop footpoints before fading. The propagation speed of the wave was about $460-510 \mathrm{~km} \mathrm{~s}^{-1}$, which is very close to the sound speed at the temperature obtained from the DEM analysis. Fang et al. (2015), using a 2.5D MHD simulation and forward modeling, reproduced such slow wave reflections in a hot coronal loop. A flare-like instantaneous energy perturbation at the footpoint evaporates a plasma blob which then propagates as a slow wave front. The wave then bounces back and forth along the loop as observed by Kumar et al. (2013). Fang et al. (2015) also used line parameters of the synthesized SUMER Fe line to show that these are propagating modes. With the use of a particle tracer, these authors confirm that such PDs better agree with a dominant wave scenario along with a mass flow component. Recently Kumar et al. (2015) reported quasi-periodic intensity oscillations in AIA EUV channels with the X-ray channel of a Fermi gamma-ray burst monitor. These authors also proposed a repetitive reconnection scenario on a fan-spine magnetic topology to explain the observed periodicity.

In this paper, we report four observations of reflective longitudinal waves seen with the HINODE/X-ray Telescope (XRT). One such event is also simultaneously observed by $S D O / A I A$. We present the observations and the analysis of the events in Sections 2 and 3. The generation mechanisms of the observed waves for each of the individual events are discussed in Section 4. The numerical setup and the forward modeling is described in Section 5 and the analysis of the model output data is described in Section 5.1. Finally we summarize and conclude in Section 6.

\section{OBSERVATION AND DATA REDUCTION}

The datasets used in this study are obtained from different active regions observed by the XRT (Golub et al. 2007) on board HINODE (Kosugi et al. 2007) and the AIA (Lemen et al. 2012), onboard SDO (Pesnell et al. 2012).

The XRT data are calibrated using xrt_prep.pro (available in the SolarSoft package; Freeland \& Handy 1998) which performs the correction for near-saturated pixels, removal of spikes, correction for contamination spots and removal of the

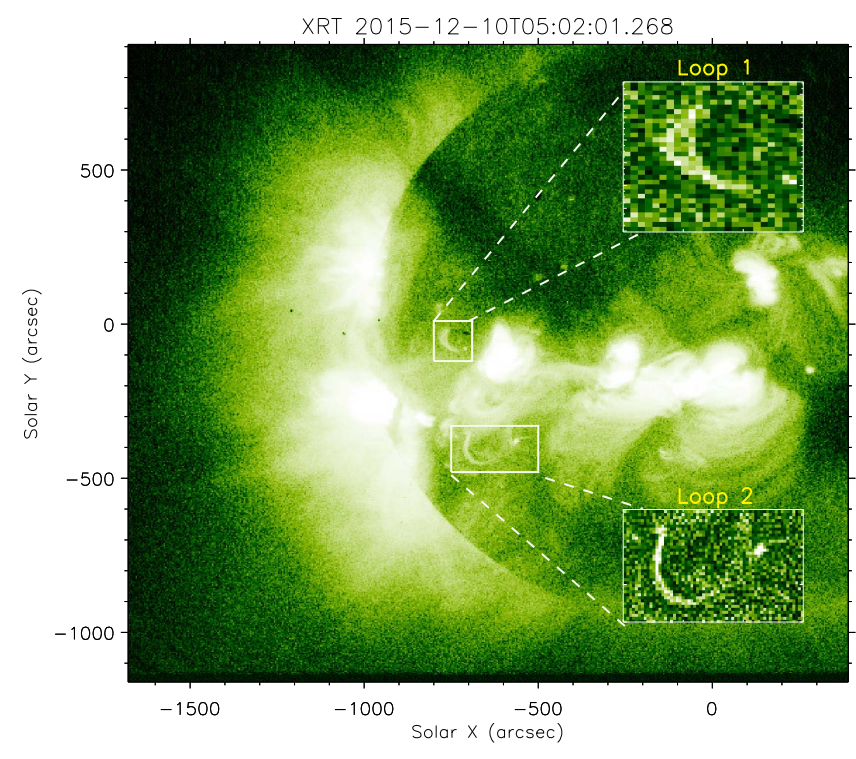

Figure 1. Context image showing the full field of view of the observation on 2015 December 10. Two white boxes highlight our loops of interest. The zoomed-in view of these individual boxes are also plotted on top of the image. (An animation of this figure is available.)

CCD bias and dark current. The final pixel scale and the cadence for each XRT observations are given in Table 1.

The AIA level 1.0 data have been reduced to level 1.5 using the aia_prep.pro which makes the necessary instrumental corrections. The final pixel scale, in both $X$ and $Y$ directions, is $\approx 0.6^{\prime \prime}$. The cadence is $12 \mathrm{~s}$.

\section{DATA ANALYSIS AND RESULTS 3.1. XRT Data Analysis}

\subsubsection{Observation on 2015 December 10}

We used HINODE/XRT data taken in the Be-thin filter on 2015 December 10. Figure 1 shows the context image of the observation along with the two loops, (loop 1 and loop 2) from an active region AR 12465, which show reflecting wave propagation.

From the movie (movie 1, available online) we see that the onset of the propagating intensity disturbance is caused by a flare which occurred at one of the footpoints. The disturbance then reflects back and forth a couple of times before fading. To see the propagation of this perturbation through the loop, we stack different snapshots of loop 1 in Figure 2 where one full reflection is seen clearly. We scaled each image to enhance the propagating intensity for better visualization. Speeds of these perturbations are estimated using the time-distance maps. We put artificial slits tracing the loops to create the time-distance 


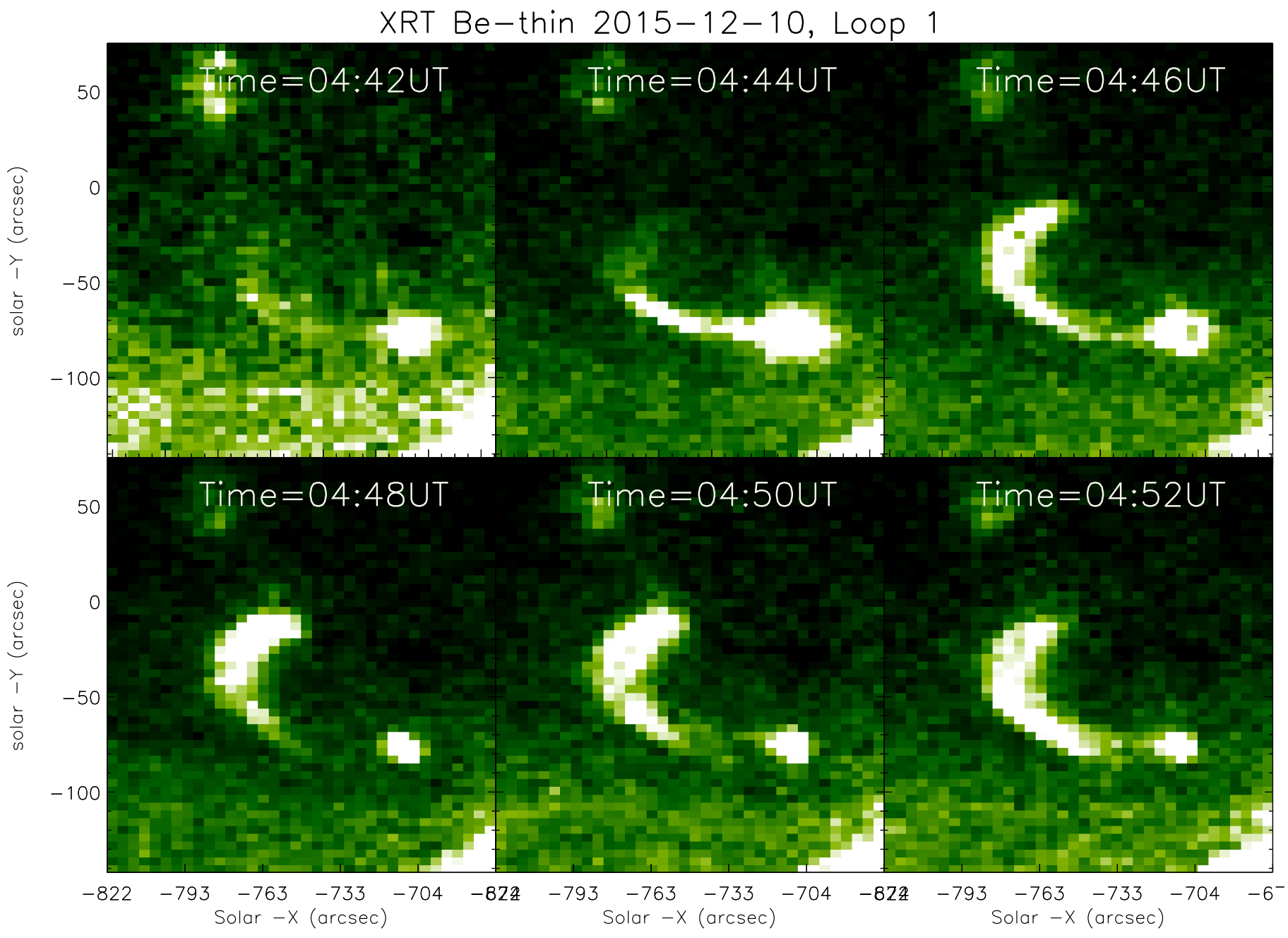

Figure 2. Sequence of base difference images for loop 1. The bright intensity perturbation starts from one footpoint at 04:42 UT and then reflects back from the other footpoint at 04:52 UT.

maps. Figure 3 shows the artificial slit positions and the timedistance maps, created using a base difference image sequence, for both loop 1 and loop 2 .

For loop 2 (panel (a) in Figure 3) we see a single reflection within the duration of the observation. The period obtained from the time-distance map (panel (b) in Figure 3) is $\approx 20$ minutes. The slopes of the bright ridges are an estimate of the speeds and we obtained a speed of $433 \mathrm{~km} \mathrm{~s}^{-1}$ and $257 \mathrm{~km} \mathrm{~s}^{-1}$ for ridges 1 and 2 respectively. For the second loop (loop 2, panel (c) in Figure 3) we see two clear reflections before the signal faded. The estimated speeds for ridges 3-5 are $391 \mathrm{~km} \mathrm{~s}^{-1}, 219 \mathrm{~km} \mathrm{~s}^{-1}$, and $251 \mathrm{~km} \mathrm{~s}^{-1}$ respectively. Loop lengths obtained by tracing the loops are 172 and $61 \mathrm{Mm}$ for loop 1 and loop 2 respectively. These loop lengths are projected lengths and thus can be an underestimation of the actual lengths.

As we see from the time-distance maps and also from the movie, the intensity amplitudes become damped as they propagate along the loop. To obtain a quantitative measure of the damping we choose a box as shown in panel (a) in Figure 4 to see the evolution of the averaged box intensity with time. The box is chosen close to the footpoint to avoid the line-ofsight (LOS) integration effect due to the loop orientation and also to obtain a good signal-to-noise ratio near the footpoint.
The averaged box intensity is plotted in panel (b) where each peak corresponds to one reflection.

We see that with each reflection the amplitude is decreasing. A small trend, as obtained by smoothing the original curve with an increasing window size between 3 and 6 points (i.e time frames), is subtracted from the original curve (panel (b)) to produce the detrended curve shown in panel (c) in Figure 4. To measure the period and the decay time, we first do a 20 fold spline interpolation of the original detrended curve, shown as the red solid line in panel (d), to produce a smooth curve. Then we fit the interpolated profile with a damped sinusoidal function of the form

$$
I(t)=A \sin \left(\frac{2 \pi t}{P}+\phi\right) \exp \left(\frac{-t}{\tau}\right)
$$

where $A, P, \tau$ and $\phi$ are the amplitude, period, decay time and phase respectively. The best fitted curve is shown as the blue solid line in panel (d) of Figure 4. The estimated period is 10.1 minutes whereas the damping time is 10.6 minutes.

\subsubsection{Observation on 2013 January 22}

We used XRT data taken in the Be-thin filter on 2013 January 22 from 8:30 UT to 9:29 UT. The full field of view 

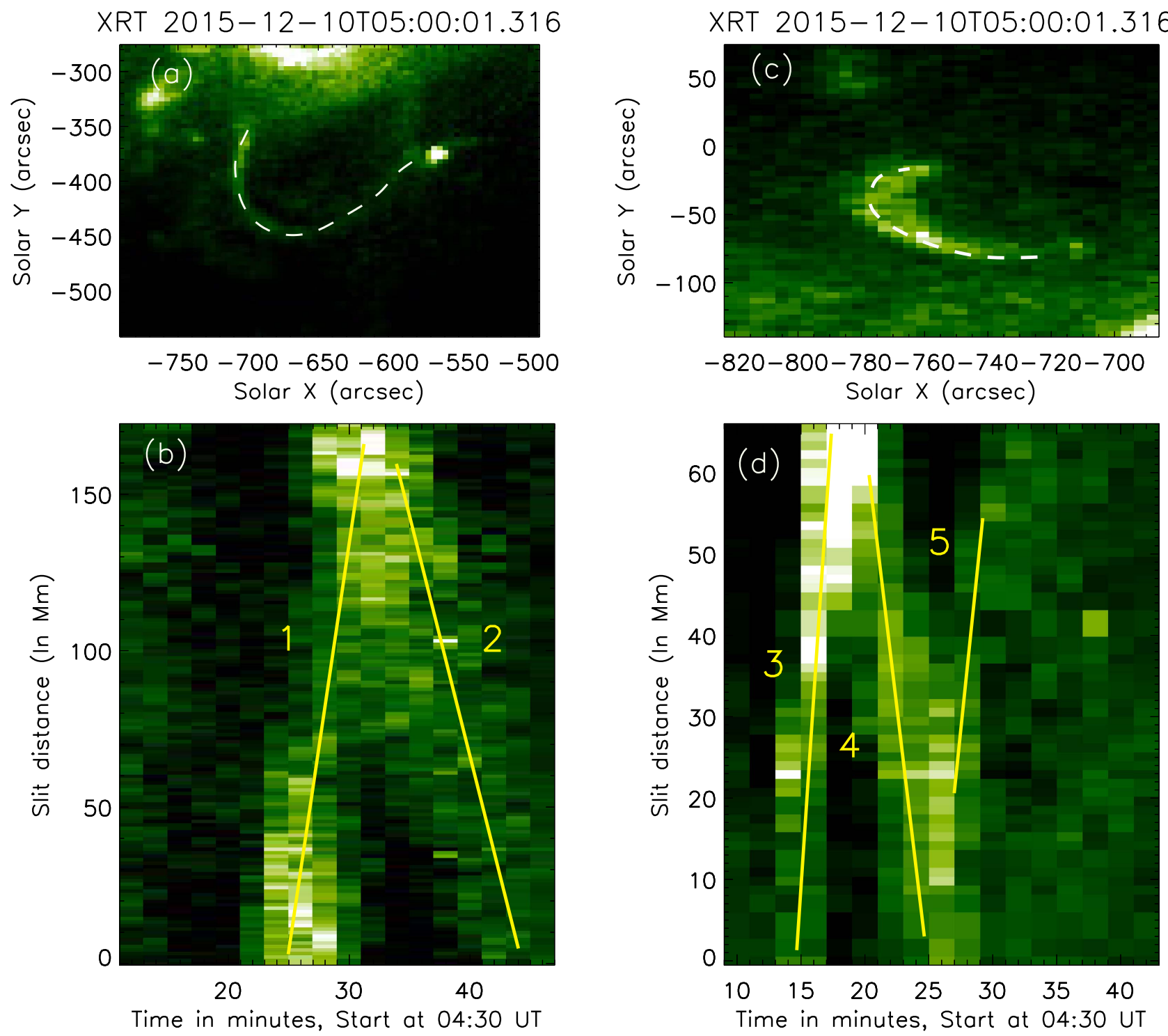

Figure 3. (a)-(b) Snapshot of loop 2 and the obtained histogram equalized time-distance map. The dashed lines show the slits used to generate these maps. (c)-(d) Same as before but for loop 1 . The yellow solid lines (1-5) in both time-distance maps highlight the slopes of the ridges.

(FOV), shown in panel (a) in Figure 5, is 394" $\times 394^{\prime \prime}$. The loop in our study is located in the active region AR NOAA 11654 in the westward solar limb. We further selected a region of interest (ROI) where the loop is seen clearly and all the analysis has been done on this selected region (panel (b) in Figure 5).

The wave appearing from one of the loop footpoints (located on the far side of the limb) at 08:59 UT is reflected from the other footpoint and travels along the loop before fading away. To see the propagation of the wave clearly, running difference images have been created as shown in panels (a)-(h) in Figure 6. We see the onset of the propagation at 08:59 UT and we mark it with a white arrow (panel (a) in Figure 6). We also mark the position of the intensity enhancement in the previous frame, seen as a black region following the white, with yellow arrows. At 9:07 UT the wavefront reaches the other footpoint and is reflected back from there. We have estimated the projected loop length to be $142 \pm 3 \mathrm{Mm}$ by tracing the points along the length of the loop ("+" signs in Figure 5). Using the measured loop length and the time the wavefront takes to travel from one footpoint to the other (08:59 UT to 09:07 UT), we have an estimate of the average speed of wave propagation and it is $\sim 295 \mathrm{~km} \mathrm{~s}^{-1}$.

To measure the propagation speed more accurately we have created time-distance map from the running difference image sequence, as shown in panel (i) in Figure 6. The time-distance map has been created using an artificial slit which traces the loop (white "+" signs in panel (b) in Figure 5 represent the slit position) and extends the slit width by two pixels across to increase the signal-to-noise ratio. From the map we clearly see the reflection of the wave from the other end of the loop and then the wave propagates back along the loop before fading around midway. The slopes of the ridges represent the propagation speeds. Different slopes of the ridges in the time-distance map reflect the change of the loop orientation with the LOS as the wave propagates along the loop. This event was also co-observed by the "Ti-poly" filter, but neither the loop nor the intensity disturbance was seen in this channel. 

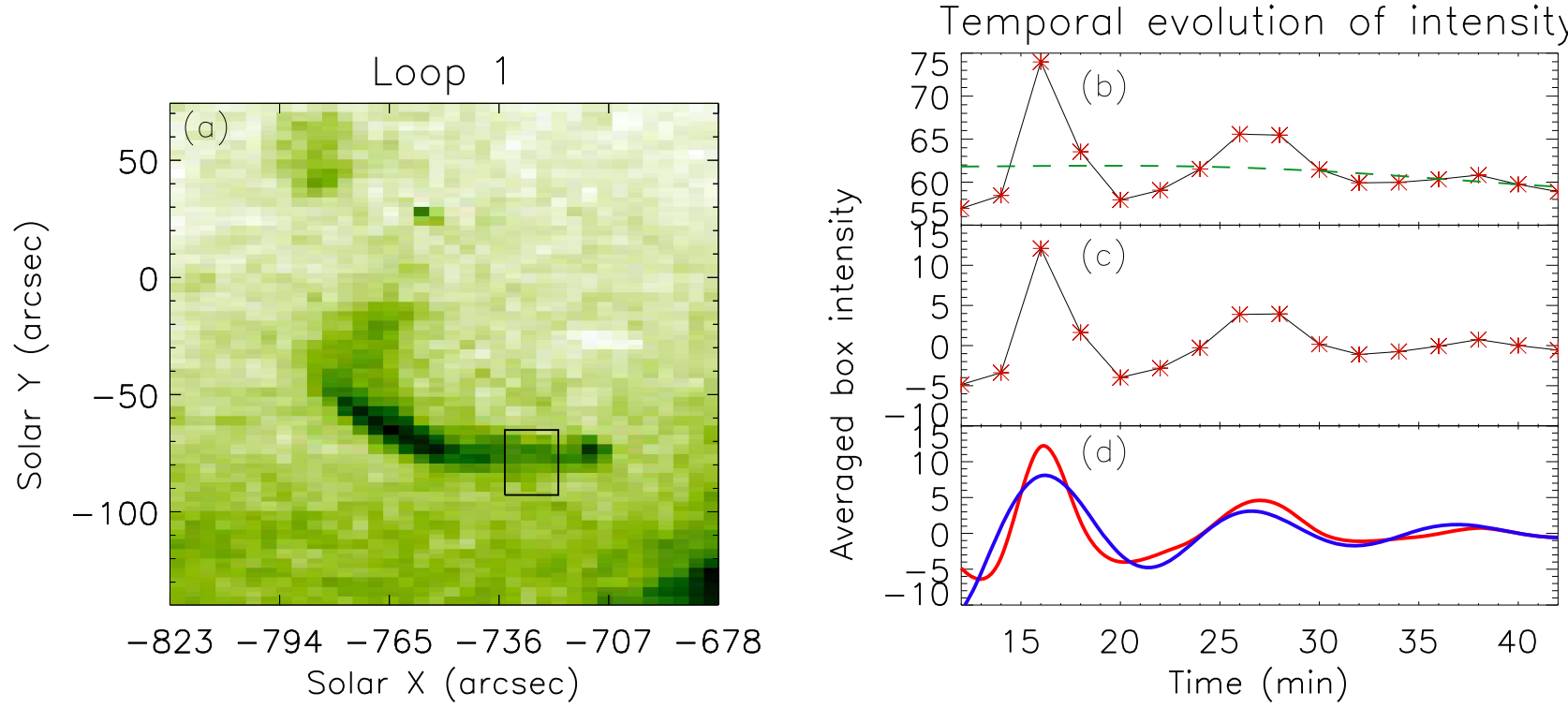

Figure 4. (a) Snapshot of the region showing the loop structure (in inverted color). The black box outlines the region selected to extract the intensity. (b) Temporal evolution of the averaged intensity over the box. The small trend, highlighted with a dashed green line, has been subtracted from the original curve to produce the detrended light curve as shown in panel (c). (d) The interpolated light curve is shown with the red line and the fitted decaying sinusoidal is shown as the blue solid line. The start time of these profiles is 04:30 UT.

(An animation of this figure is available.)
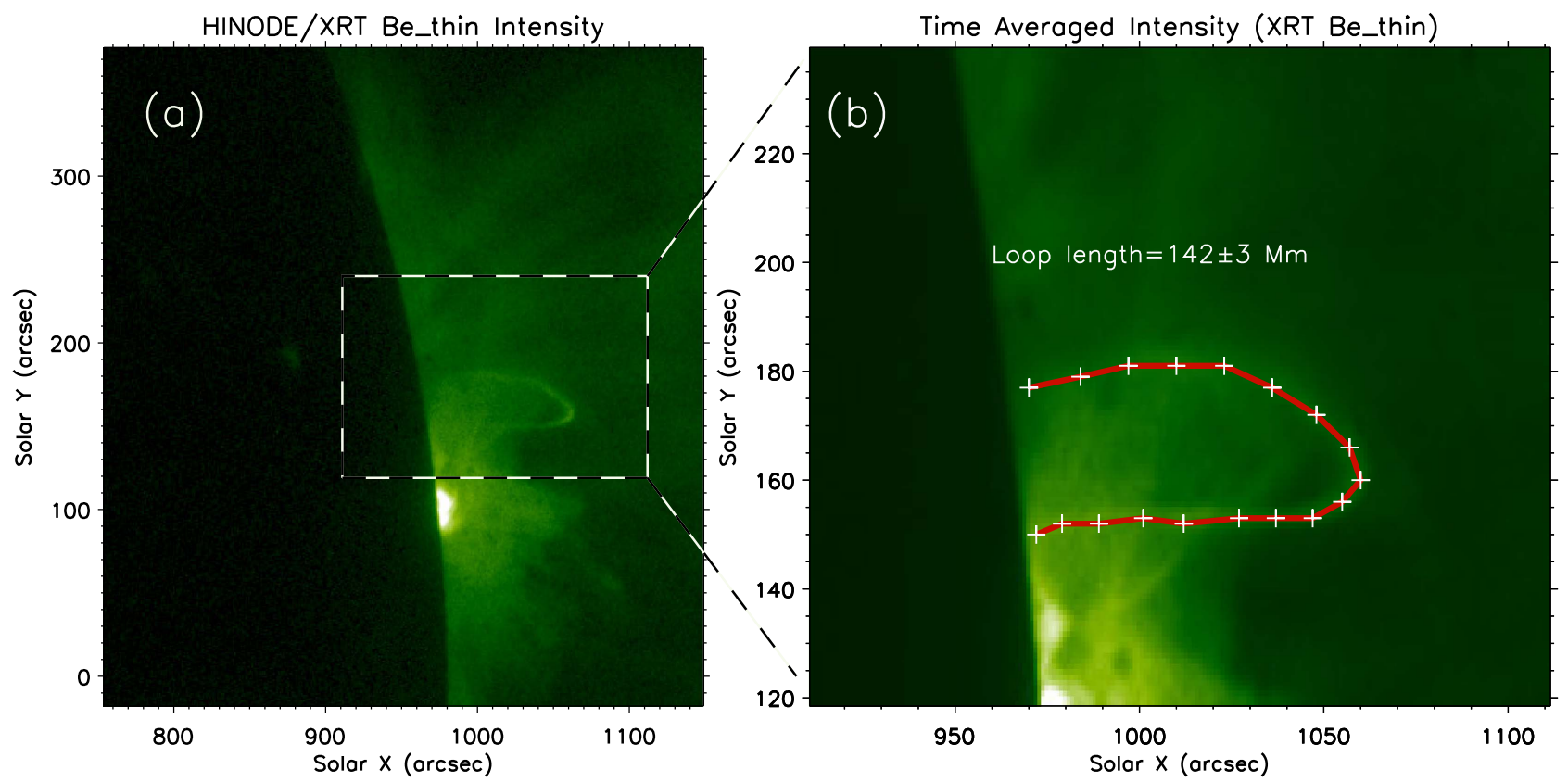

Figure 5. Panel (a) shows the full field of view of the January 22 XRT data. The black-white rectangular box shows the region of interest (ROI) selected for the analysis. A movie of the ROI is available online (Movie 3). Panel (b) shows the time-averaged image of the ROI. The loop length calculated by tracing the loop (white "+" signs) is printed on the plot. The red line is used to create the time-distance plot.

(An animation of this figure is available.)

\subsubsection{Observation on 2013 January 27}

On 2013 January 27, a similar event was observed with the XRT Be-thin filter. A flare-like brightening at one of the footpoints is the source of the wave which propagates along the loop. The top panel in Figure 7 shows the full FOV of the XRT observation and the ROI respectively. We have estimated the loop length by tracing points along the loop and the length is equal to $90 \mathrm{Mm}$ (with errors less than $5 \mathrm{Mm}$ ).
From the movie (Movie 4) we see that the wave is damped rapidly as it propagates through the loop and it fades away as soon as it is reflected from the other footpoint. This is also seen in the processed time-distance map (last panel in Figure 7) by placing an artificial curved slit following the loop as shown by the "+" signs. The processed time-distance map has been created by scaling individual time in the original map to enhance the bright pixels. In the processed time-distance map 

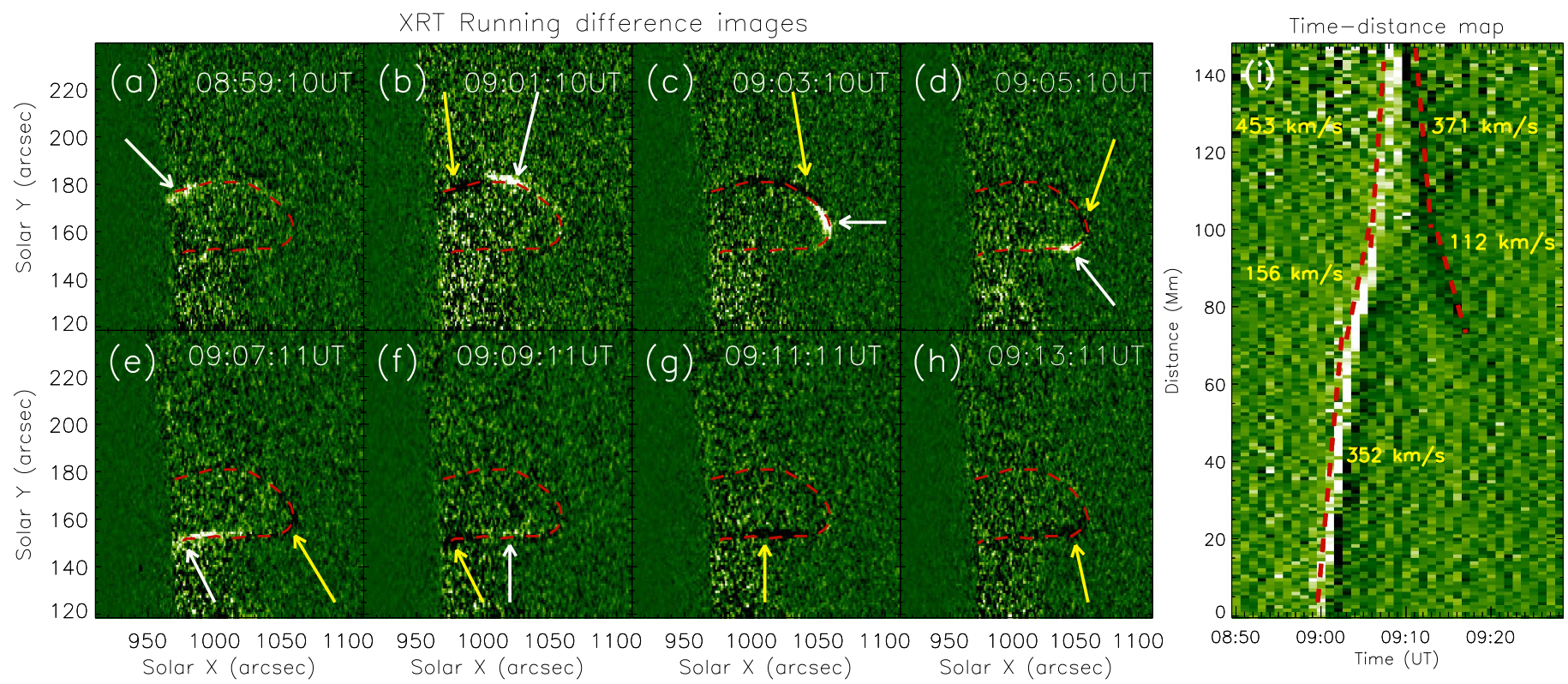

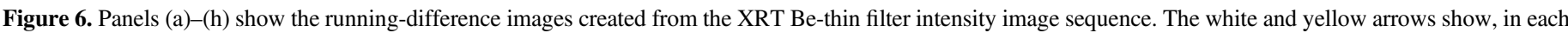

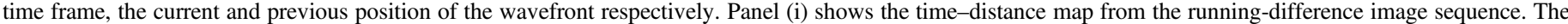
white and black inclined ridges show the forward and reflected wave propagation along the loop while the red lines indicate the slopes of these ridges.

we see a reflection signature, though not prominent, from the other footpoint before the signal drops considerably. The speed calculated from the inclined ridges (highlighted with yellow dashed lines) are 442 and $320 \mathrm{~km} \mathrm{~s}^{-1}$ respectively.

The propagation and the reflection of the wave are clearly visible in the base-difference images shown in Figure 8. Panels (a)-(h) show the intensity propagating from one footpoint to the other and in panel (i) we see the reflected part moving in the other direction.

\subsection{AIA Data Analysis}

The XRT "Be-thin" filter mostly observes the "hot" plasma (the response function of the "Be-thin" filer peaks at $10 \mathrm{MK}$ ). This leads to the fact that the event observed in this filter is likely to be captured in the hotter channels of the AIA (94 and $131 \AA$ A). Despite this, we found only one event (on 2013 January 22) where the loop is also simultaneously seen with AIA. This is because the AIA $94 \AA$ and AIA $131 \AA$ channels have a second peak around $1.5 \mathrm{MK}$ and $0.5 \mathrm{MK}$ respectively, next to their expected response at $10 \mathrm{MK}$. These secondary peaks contaminate heavily when the loop is on the disc rather than when it is on the limb. Also for the other events, the plasma may be too hot to be seen in any of the AIA channels. It must be mentioned here that although we did not see the loop (except for the event on 2013 January 22), we have captured the time evolution of the loop footpoints for all the other events. For the event on 2013 January 22 we could not locate the footpoint as it was on the far side of the Sun.

For the event on 2013 January 22, we found that the loop is also detected in the AIA $94 \AA$ channel (only), indicating the presence of a high-temperature plasma. We have used the corresponding SDO/AIA data in the $94 \AA$ channel taken from 8:30 UT to 9:30 UT. We used this data to co-align the two instruments and the final XRT coordinates are obtained after correcting for the offsets. The left panel in Figure 9 shows the time-averaged image of base-difference image sequence of the AIA $94 \AA$ channel. We also estimate the loop length in a similar way as the XRT measurement and the length is equal to $137 \pm 2 \mathrm{Mm}$. From the movie (movie 5, available online) we clearly see that the wave starting from one footpoint gets reflected from the other, and fades away after traveling a certain distance along the loop-length. This feature is also seen clearly from the time-distance map created from the AIA basedifference image sequence. Two oppositely inclined ridges are visible in the map, indicating a clear reflection signature. We also estimated the propagation speed from the slope of the ridges and they are shown in Figure 9.

\subsection{DEM Analysis}

Wave propagation through a loop largely depends upon the physical parameters of density, temperature etc. Since the sound speed in a medium depends on its temperature, we performed automated temperature and emission measure analysis, using a SolarSoft code, as developed by Aschwanden et al. (2013) to estimate average density values and temperature inside the coronal loop.

In order to estimate the DEM, the SolarSoft code co-alignes AIA images in different EUV passbands (94, 131, 171, 193, 211,335 A) using a solar limb fit. Using the forward fitting of the observed DEM distribution with that of the model DEM distribution, the peak emission measure and the peak temperature at a particular pixel are determined. In our case, the loop is only poorly visible in AIA $94 \AA$ channel. Thus the density and temperature values, obtained from the DEM analysis, are only rough estimations for both parameters. This order of magnitude estimate is sufficient for our purpose as we insert these values in our numerical model (described in the following section) as initial loop parameters. To check for any spatial as well as temporal evolution of the loop parameters, we performed DEM analysis at three different locations at three different times. One such case is shown in detail in Figure 10. The estimated average loop density and temperature are $\approx 10^{9} \mathrm{~cm}^{-3}$ and $\approx 10 \mathrm{MK}$ respectively. Using this temperature value, the sound speed within the 


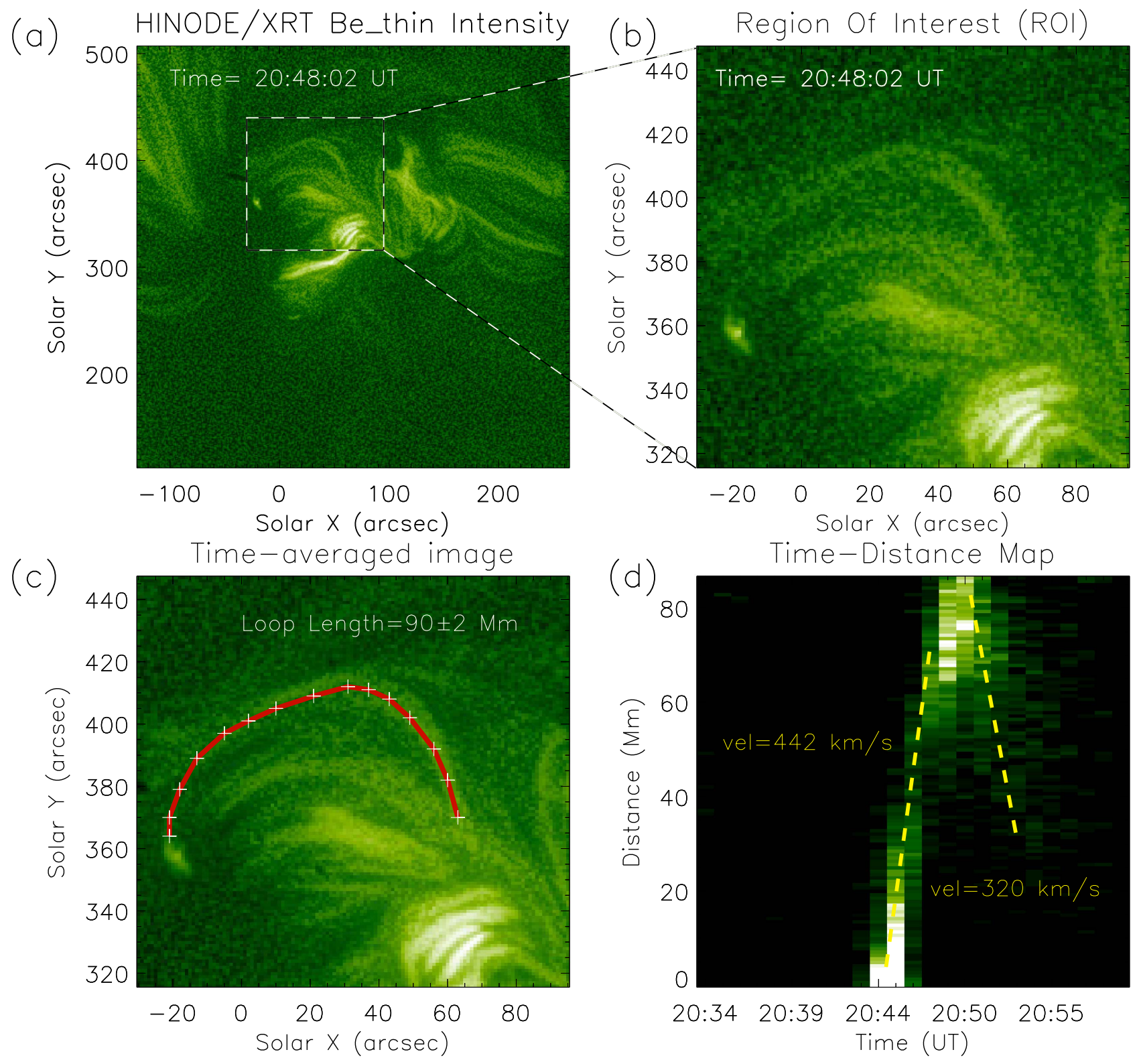

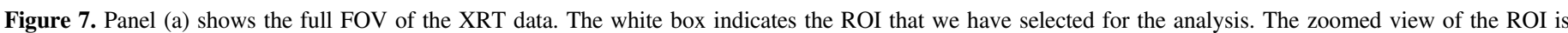

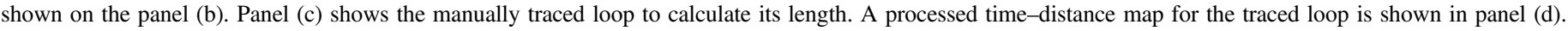
Estimated speeds are printed on the panel.

(An animation of this figure is available.)

loop is $\mathrm{c}_{s} \sim 152 \sqrt{T(\mathrm{MK})} \sim 480 \mathrm{~km} \mathrm{~s}^{-1}$. The other two DEM measurements are shown in Figure 11.

\section{GENERATION MECHANISM}

The generation mechanism of such waves is not yet fully understood. There is evidence of small (micro-) flares, occurring at the loop footpoints, as seen in our events also, as being the generator of the waves. See Wang (2011) for a complete review on this.

In the event on January 22, the flare occurred at the far side of the Sun and thus it was hidden from us (STEREO also did not capture the event due to a coarser cadence of 5 minutes). We did capture the loop footpoint for the event on January 27 from the XRT as well as from the AIA EUV channels. Figure 12 shows the time evolution of the loop footpoint as seen with the XRT. We notice a complex mini-loop-like structure at the footpoint with many spines. We also notice that an intense brightening which starts at the right end of the loop footpoint (panel (c)) moves toward the left in the next time frame (panel (d)). This observed occurrence and evolution of the brightening at the loop footpoint resembles very much the "blowout-jet" examples found previously (Moore et al. 2013).

Although the loop is not visible in any of the AIA channels, the loop footpoint is clearly visible in all the EUV channels. Figure 13 shows the time evolution of the footpoint in five EUV channels of the AIA (171, 131, 94, 193, $304 \AA$ ). Panels (a)-(e) show the snapshots of the footpoints at the time when the flare peaks. We see a complex loop arcade with a spine forming at the top of the structure. From the movie (movie 6) we also notice that the AIA cooler channels (171 and $304 \AA$ ) along with a hotter channel $(193 \AA$ ) capture a small "filament- 
XRT Base Difference Images

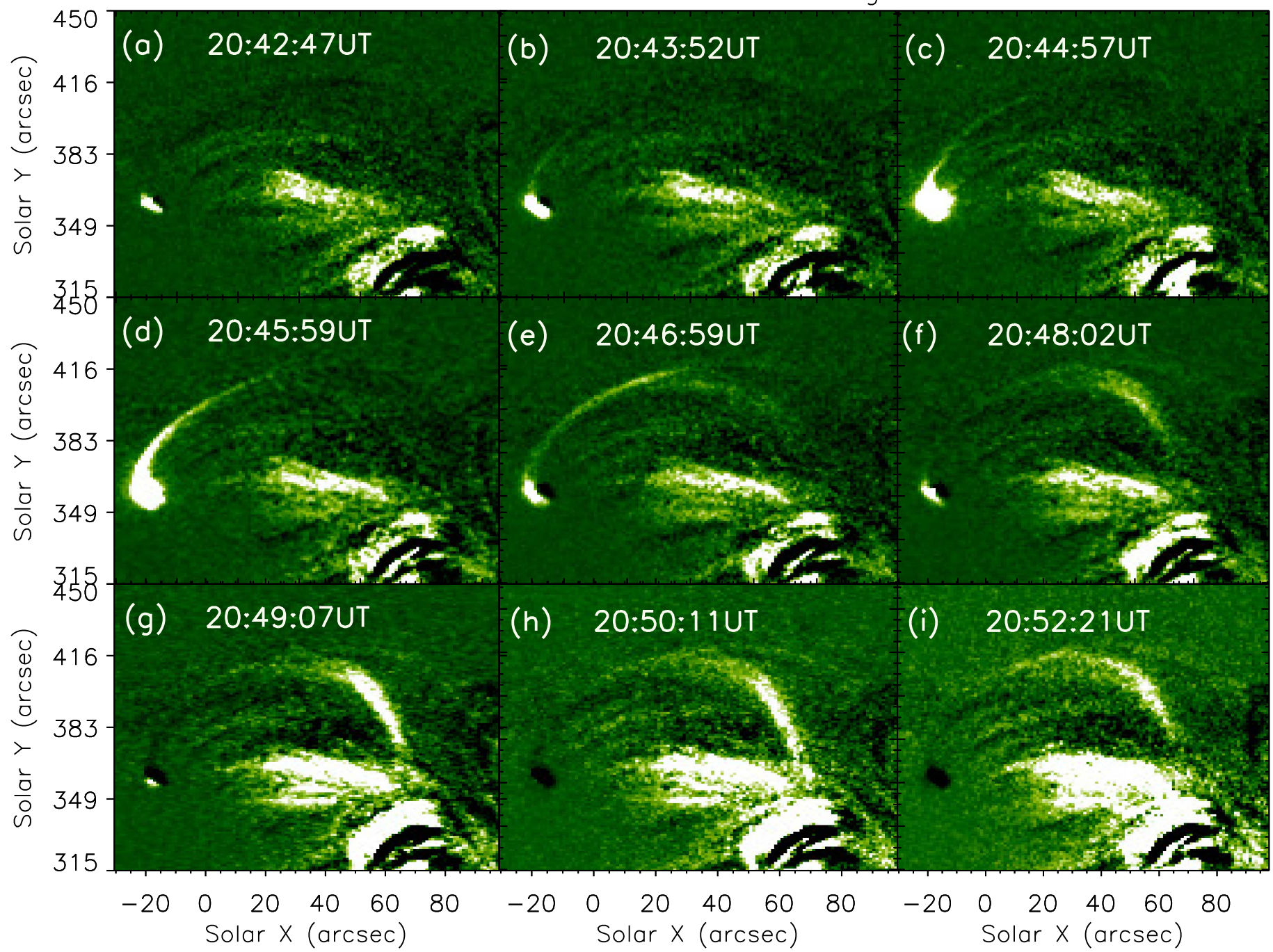

Figure 8. Base difference XRT images of the ROI, showing the wave propagation from one loop footpoint to the other. Each image is scaled individually to highlight the wavefront.

like" dark feature rising with the evolution of the flare (Sterling et al. 2015). However, we must emphasize that the initial location of the filament before the flare is not clearly visible. To see the photospheric magnetic filed configuration associated with this structure, we overlay the Helioseismic and Magnetic Imager (HMI) LOS magnetic filed contours $( \pm 20 \mathrm{G})$ on top of the AIA images. We do not see a clear bipolar structure around the footpoint region in this case, suggesting that the reconnection might have happened higher in the atmosphere. Panels (f)(j) in Figure 13 show the snapshots of the same region when the ejecta is seen to propagate through the spine. Though the ejected material is poorly visible in the AIA $94 \AA$ channel, it shows up in the rest of the channels. A yellow rectangular box, which marks the footpoint region in panels (a)-(e), is also overplotted in panels (f)-(j) for better comparison.

The footpoint structure almost disappeared at this moment (panels (f)-(j)) leaving the spine through which the ejected plasma moves. To measure the propagation speed of the plasma in the plane of sky, we create the time-distance maps for all the channels by placing an artificial slit as indicated by a white curved rectangle in panels (f)-(j). The obtained time-distance maps for individual channels are plotted in the bottom panel of
Figure 13 (panels (k)-(o)). Here we see two slanted ridges in all channels except the $304 \AA$ channel where the second ridge is not visible. The speeds calculated from the slopes of the first ridge in all the cases range from 122 to $139 \mathrm{~km} \mathrm{~s}^{-1}$.

The occurrence of this ejecta coincides with the flare peak time (at 20:45 UT) as seen from the XRT. The second ejecta, which occurred $\approx 2.5$ minutes later, propagates with a much faster velocity than the first. The measured speeds in this case are 384 and $354 \mathrm{~km} \mathrm{~s}^{-1}$ in the 171 and $193 \AA$ channels respectively.

To understand the evolution of the observed footpoint intensity and its association with the photospheric magnetic field, we plot in Figure 14 the temporal evolution of the intensities from the AIA and XRT and the unsigned HMI magnetic flux averaged over the yellow box shown in panel (a) of Figure 13. A GOES soft X-ray flux (1-8 $\AA$ ) is also in the plot. We see a clear association of the XRT intensity peak with intensity increments in all the AIA EUV channels. This increment is accompanied by a soft X-ray flux enhancement. A closer investigation on the AIA light curves shows another peak, approximately 1 minute later, with smaller magnitude than the first. It is worth mentioning here that the second peak 


\section{3-01-22 SDO/AIA}
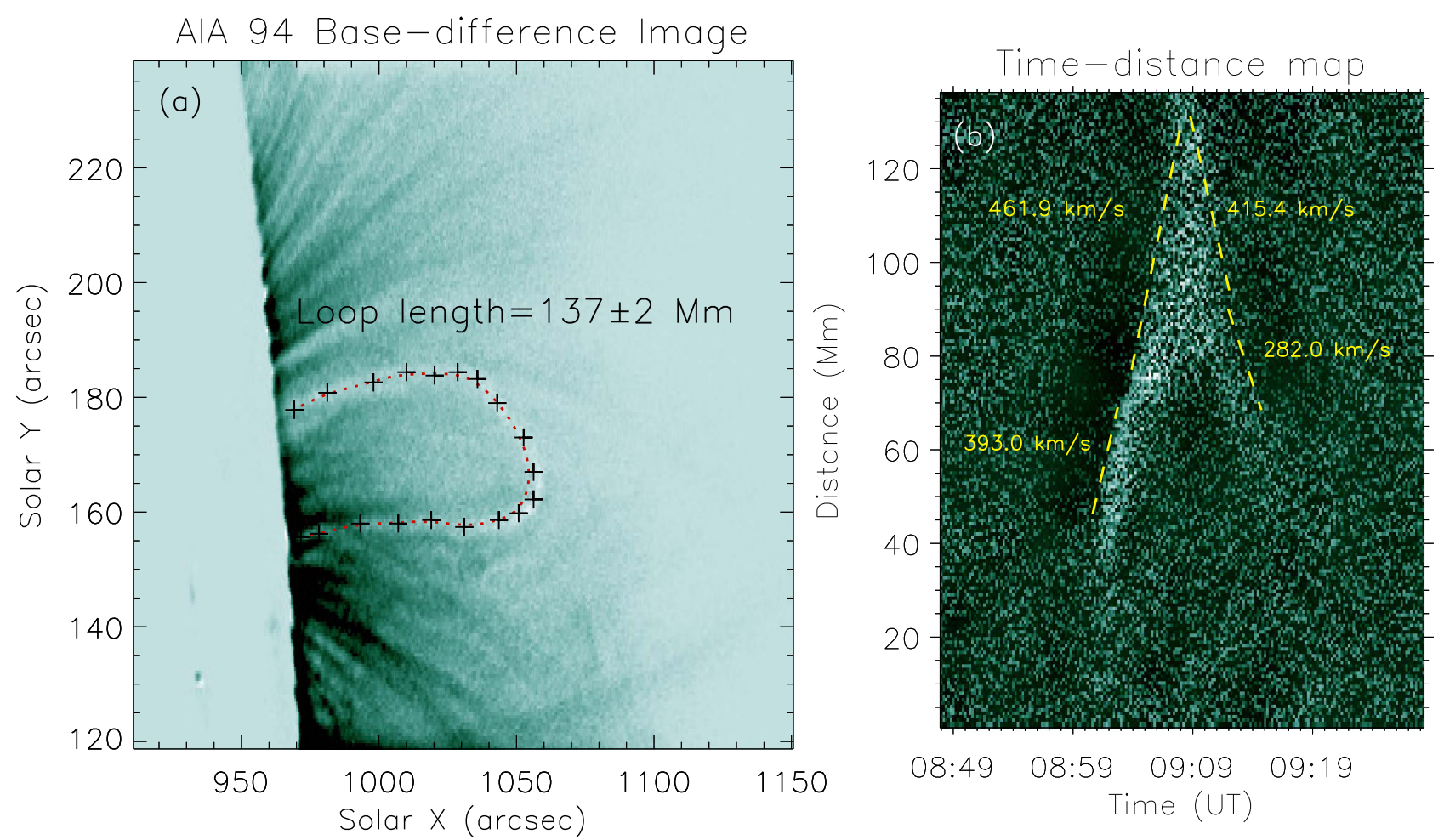

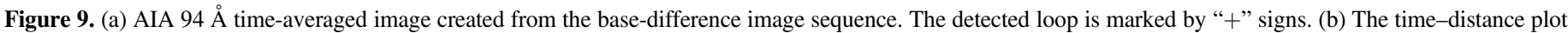
showing two oppositely inclined white ridges. Yellow lines indicate the slope of these ridges.

(An animation of this figure is available.)

is very weak in the $94 \AA$ channel and there is no such peak seen in the XRT intensity prfile. The absence of the second peak in the XRT profile can be explained by considering the coarser time and spatial resolution of the XRT compared to the AIA and also the filter response of the XRT "Be-thin" filter. In the last panel of Figure 14 we plot the total (unsigned) flux from the boxed region and we notice that there are two dips on the profile around the flare time (though these dips are not significantly strong, and are comparable to the fluctions seen in the time series). Note that the second ridge, found in the timedistance maps in Figure 13, originates due to the second peak found in the intensity profiles of the AIA. We propose a scenario where the chromospheric plasma becomes heated rapidly due to the flare and produces the high-speed ejecta which is seen to propagate in all the coronal channels. Here we want to highlight that there is a delay of $\approx 1.5$ minutes between the second intensity enhancement and occurrence of the highspeed plasma. Such a scenario of rapid heating of the chromospheric material also explains the absence of this second ejecta in the chromospheric $304 \AA$ channel.

Now we analyze the event that occurred on 2015 December 10. The XRT observed this event with $4 \times 4$ spatial binning, resulting in a spatial scale of 4!" 1 in both $x$ and $y$ directions. Thus we could not resolve the footpoint from the XRT data (the cadence was also coarser in this case, $121 \mathrm{~s}$ ). Using the AIA data, we do not see the loop structure in this case either, but the footpoints (for both loop1 and loop2) are seen in all the AIA EUV channels. Panels (a1)-(e6), in Figure 15, show the temporal evolution of the loop1 footpoint in five EUV channels of the AIA. Similar to the previous event, here also we see a jet spine at one side of the footpoint. Snapshots of the footpoint for loop2 are shown in panels (f1)-(f6). In this case, we do not see a clear jet spine structure like the previous events, although we see multiple "mini-loops" at the footpoint similar to the other events. Figure 16 shows the temporal evolution of the footpoint intensities for both loops. Panel (a) of Figure 16 highlights the yellow rectangular box chosen for the intensity evolution study of the footpoint and also highlights the artificial slit (white rectangular box) used to create the time-distance maps (shown in panels (c) $-(\mathrm{g})$ in Figure 16). From the time evolution of the intensities in the different AIA channels, shown in panel (b), we notice that a major peak occurs at 04:45 UT which matches the onset of the wave propagation as seen by the XRT (panel (d) in Figure 3). Now, from the time-distance maps (panels (c)-(g)) we see that an inclined ridge appears in all the AIA channels, co-temporally with the intensity peak. The speeds of propagation, as measured from the slopes of the ridges, range between 127 and $157 \mathrm{~km} \mathrm{~s}^{-1}$. We have also overplotted the HMI LOS magnetic field contours $( \pm 30 \mathrm{G})$ in panel (a). We see the presence of both positive and negative polarities within the yellow box but the dioplar structure is not clearly visible. For the footpoint of loop2 (panel (h)), we do not see any clear jet or spine structure but the footpoint evolution of the intensities in all channels shows a peak at 04:27 UT which again matches with the onset of the wave propagation seen from the XRT (panel (b) in Figure 3). We could not use the GOES data in this case due to the presence of multiple active regions and other co-temporal events occurring on the disc (close to the event location). 
fits/aia - Loop \#94/ 0
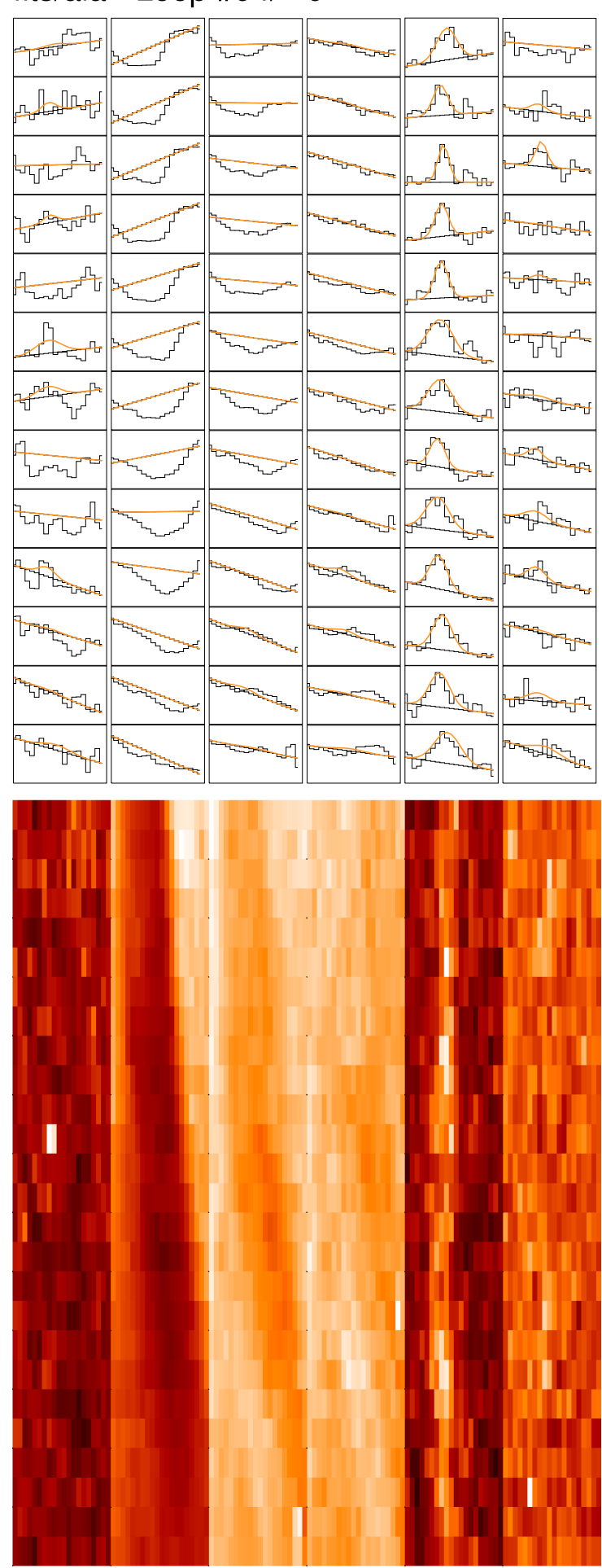

131 A 171 A 193 A 211 A 94 A 335 A
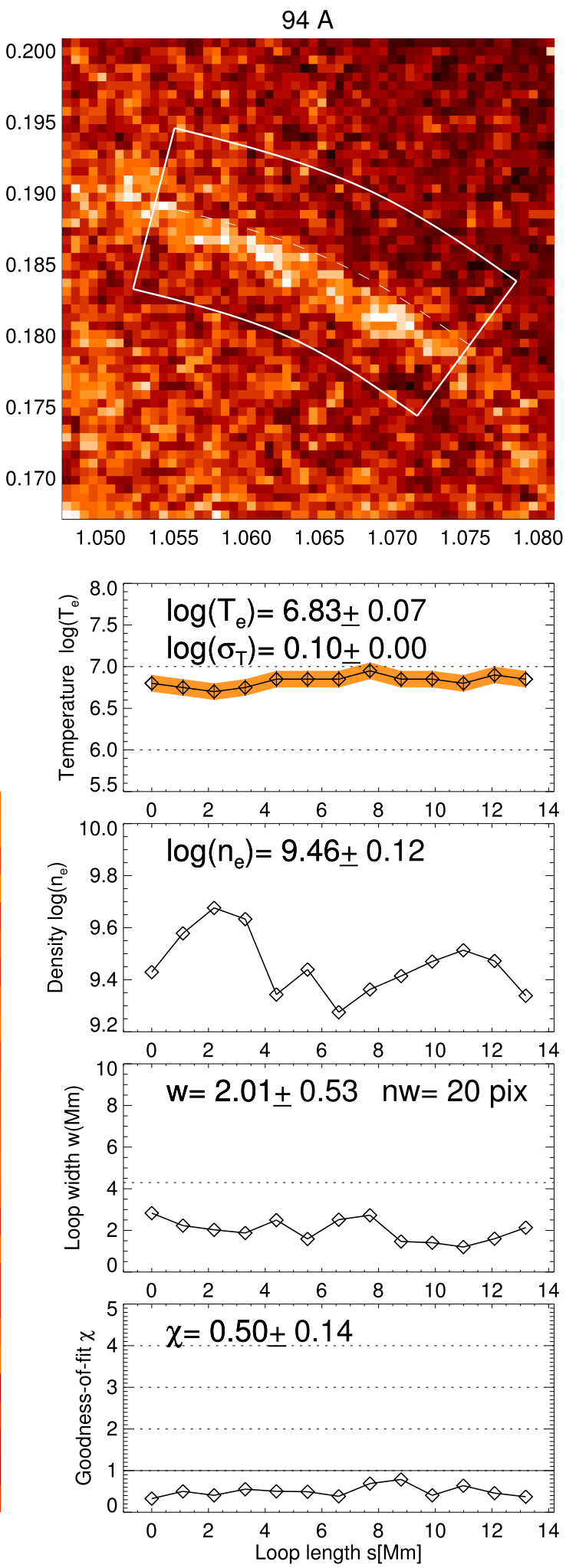

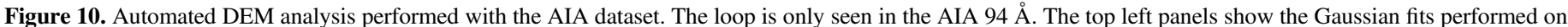

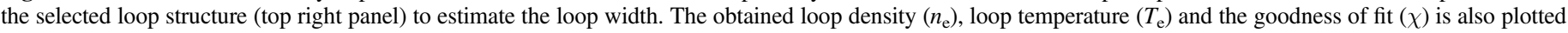
in different panels.

In summary, we see that a micro-flare at one of the footpoints of the loops acts as a trigger for the slow waves. The micro-flare originally ejects a plasma which, as soon as it becomes detached from the source, evolves as a wave packet and exhibits slow wave properties. We test our idea of this flare generating the slow waves in our numerical model (described 
$94 \mathrm{~A}$
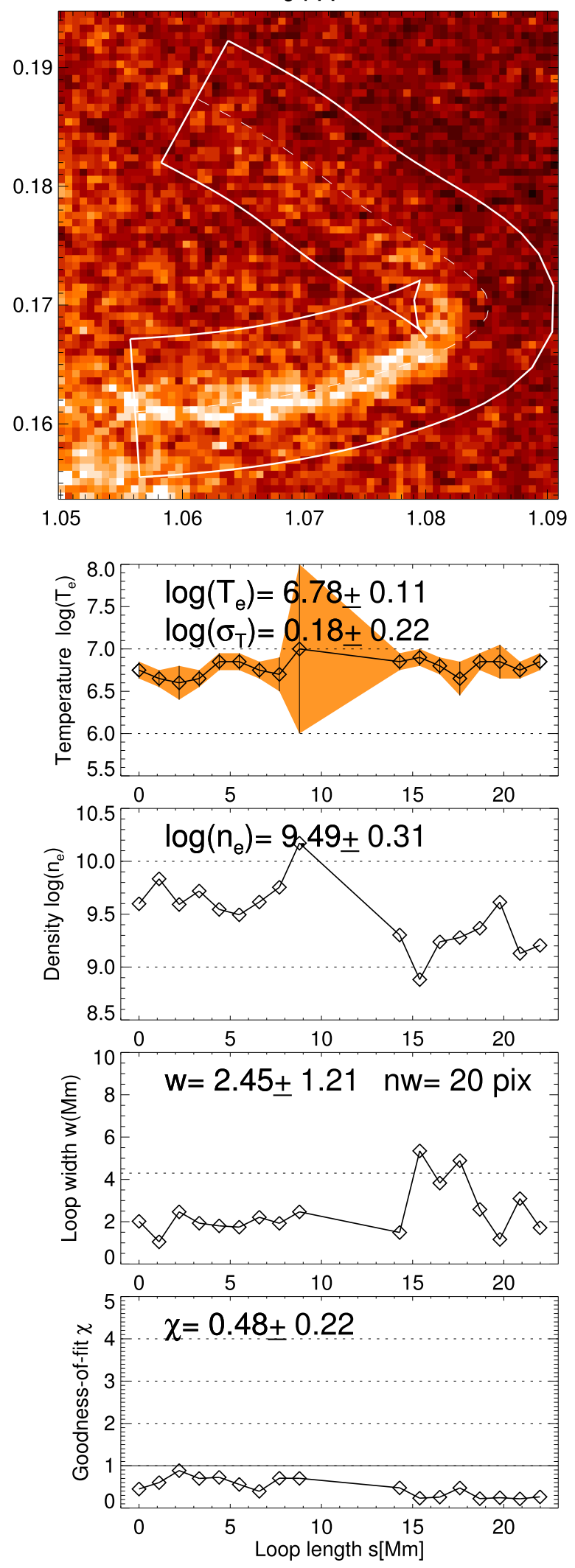

$94 \mathrm{~A}$
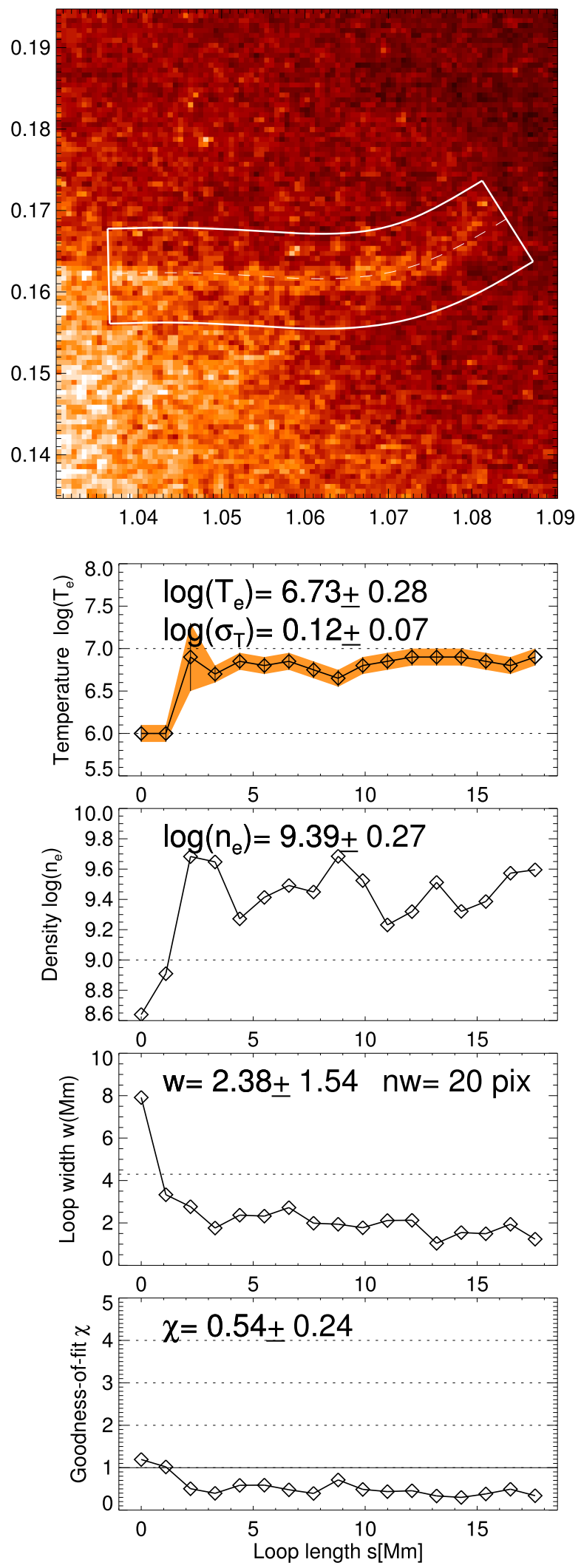

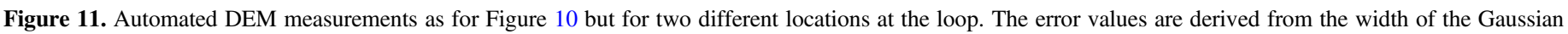
fitting (see Aschwanden et al. 2013 for details) and may be an underestimation of the actual uncertainties. 


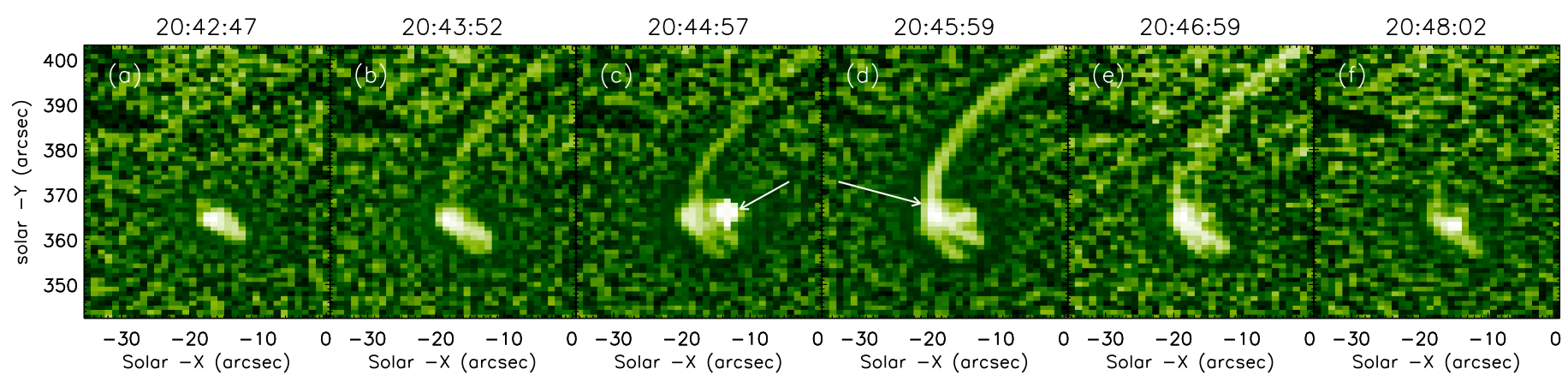

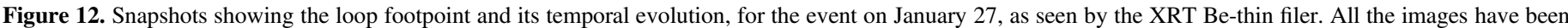
scaled to highlight the footpoint. The white arrows in panels (c) and (d) indicate the brightening position in the corresponding frames.

(An animation of this figure is available.)

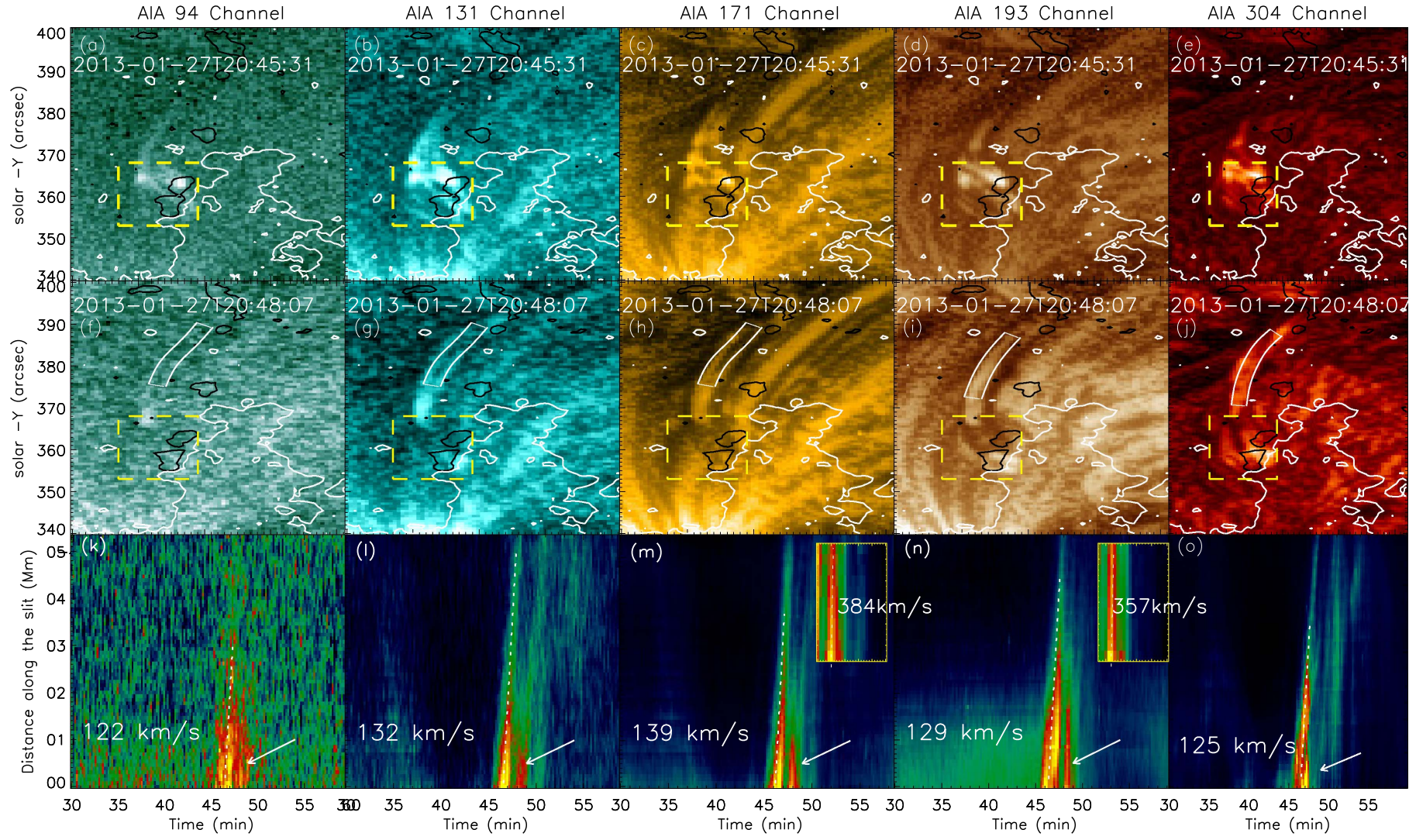

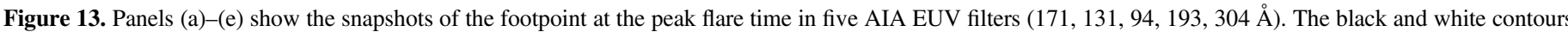

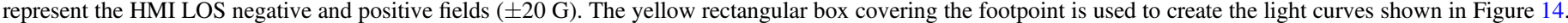

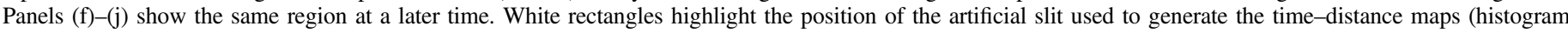

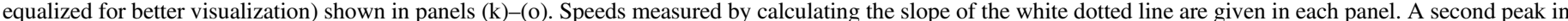

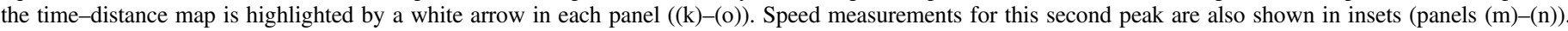
Start times of the time-distance maps are 08:00 UT.

in the next section) by injecting heat equivalent to the flare energy at one of the loop footpoints.

\section{NUMERICAL EXPERIMENT}

From observations we obtained an estimate of the speed of the wave propagating through the loop. The density and temperature values of the loop plasma are also calculated using DEM analysis using the AIA data. Now we use a numerical simulation, with the obtained loop length, density and temperature as the input parameters, to model the observations. Our simulation uses a 2.5D thermodynamic MHD model as in Fang et al. (2015) which includes gravity, anisotropic thermal conduction and radiative cooling. The box domain in the simulation is taken as $-60 \mathrm{Mm} \leqslant x \leqslant 60 \mathrm{Mm}$ and $0 \leqslant y \leqslant 80 \mathrm{Mm}$ in order to obtain a comparable loop length of $\approx 140 \mathrm{Mm}$ as estimated from our observations. 


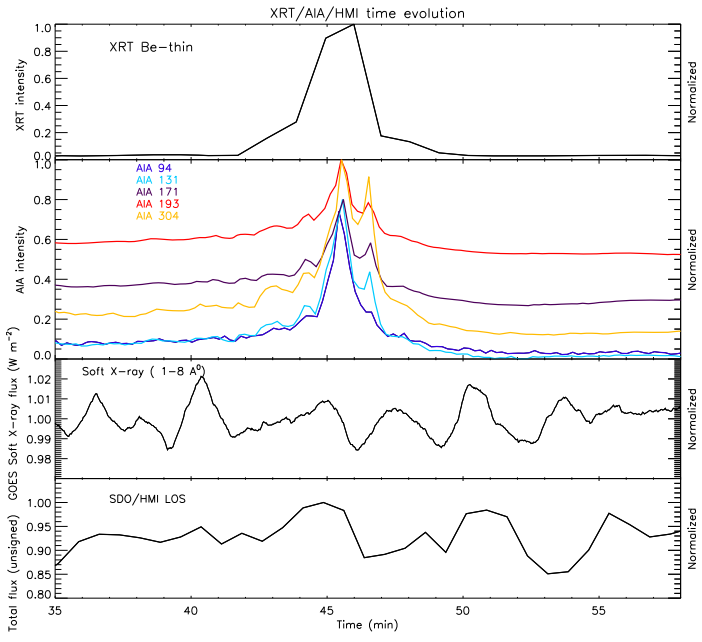

Figure 14. Top panel shows the time evolution of the box averaged intensity for the XRT data. AIA intensities for the five AIA channels are plotted in the middle panel. The bottom two panels show the light curve for the GOES soft X-ray flux (1-8 $\AA$ ) and the HMI unsigned total flux (averaged over the box). Start times of the profile are 08:00 UT.
We initialize with a linear force-free magnetic field given by

$$
\begin{aligned}
& B_{x}=-B_{0} \cos \left(\frac{\pi x}{L_{0}}\right) \sin \theta_{0} \exp \left(-\frac{\pi y \sin \theta_{0}}{L_{0}}\right), \\
& B_{y}=B_{0} \sin \left(\frac{\pi x}{L_{0}}\right) \exp \left(-\frac{\pi y \sin \theta_{0}}{L_{0}}\right), \\
& B_{z}=-B_{0} \cos \left(\frac{\pi x}{L_{0}}\right) \cos \theta_{0} \exp \left(-\frac{\pi y \sin \theta_{0}}{L_{0}}\right),
\end{aligned}
$$

with the angle $\theta_{0}=30^{\circ}$ between the arcade and the neutral line $(x=0, y=0)$ and the horizontal size of our domain setting $L_{0}=120 \mathrm{Mm}(-60 \mathrm{Mm} \leqslant x \leqslant 60 \mathrm{Mm})$ and we take $B_{0}=$ $50 \mathrm{G}$. We set the temperature below a height of $2.7 \mathrm{Mm}$ as a uniform $10,000 \mathrm{~K}$ for the initial thermal structure. The distribution of initial density is calculated based the assumption that a hydrostatic equilibrium with a number density of $1.2 \times 10^{15} \mathrm{~cm}^{-3}$ lies at the bottom of the simulation box. We assume the initial setup with a background heating rate which decays exponentially with height to approach a self-
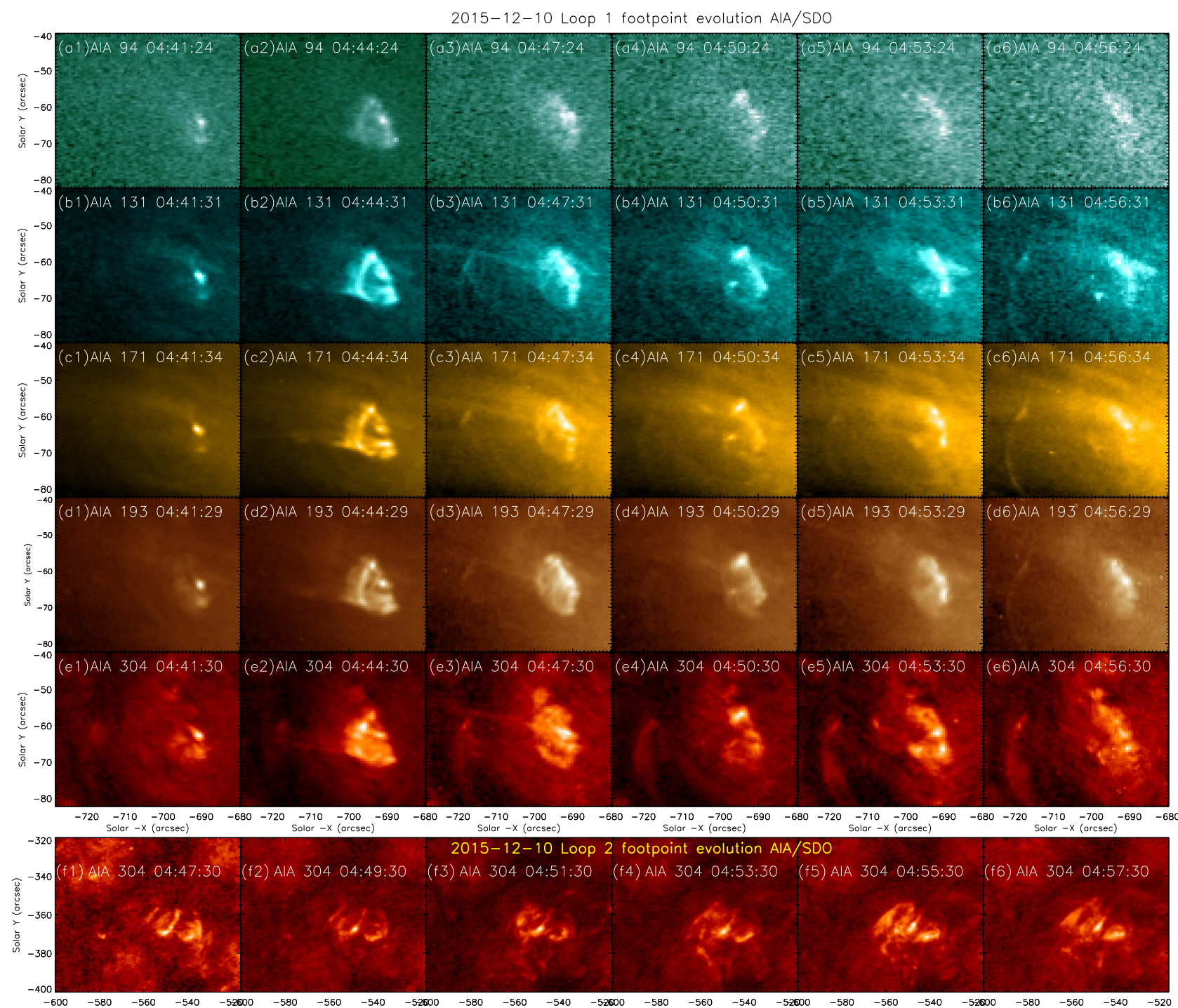

Figure 15. Panels (a1)-(e6) display the time evolution of the loop footpoint for the event on 2015 December 10 in five EUV channels during the flare time. Panels (f1)-(f6) show the same but for the footpoint of loop2. 

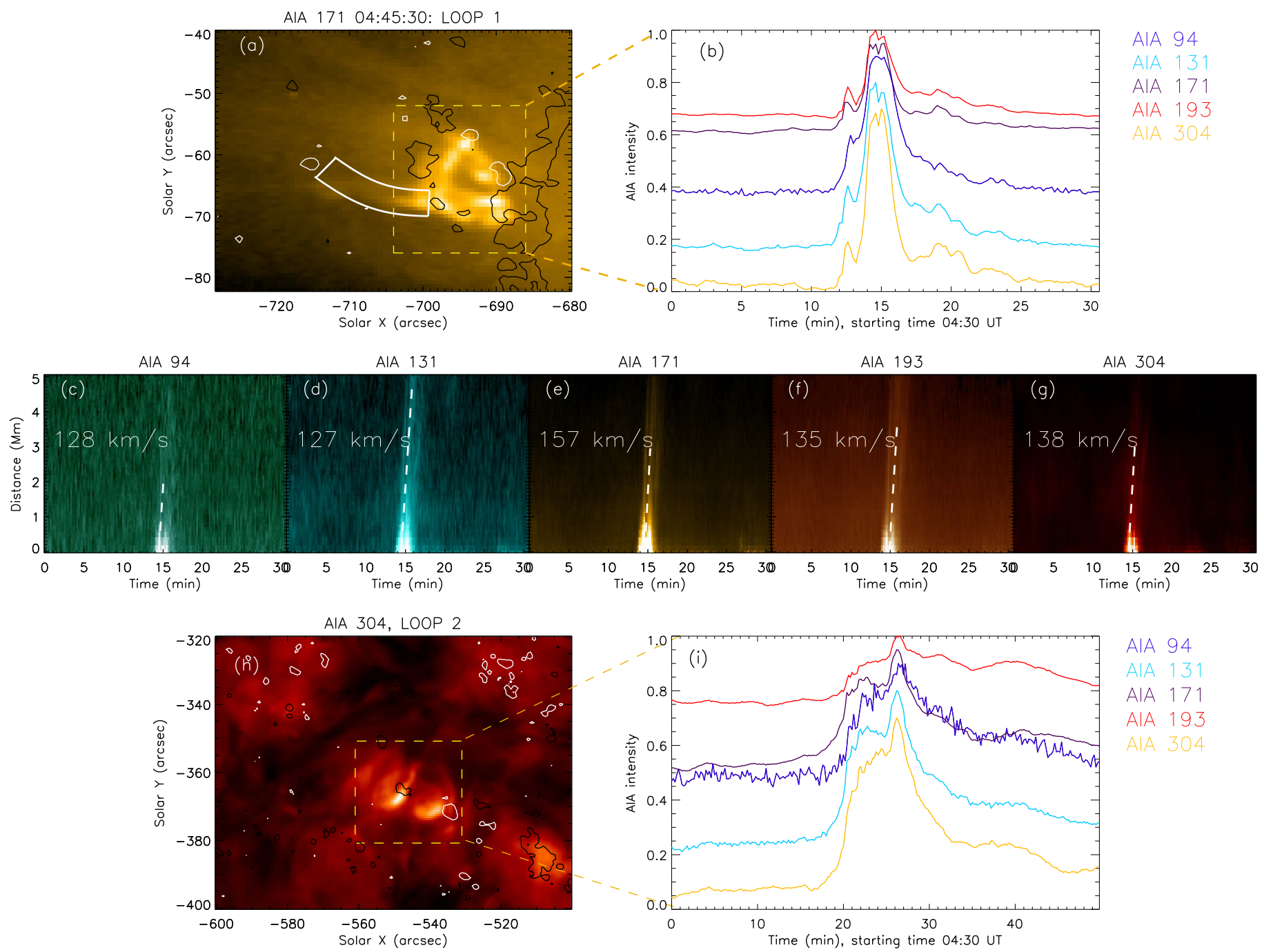

Figure 16. Panel (a) shows the snapshot of the loop1 footpoint in the AIA $171 \AA$ channel. The yellow box highlights the region used to calculate the footpoint intensity evolution in five AIA EUV channels as shown in panel (b). The white rectangular box in panel (a) marks the artificial slit used in creating time-distance maps (panels (c)-(g)). Panel (h) shows the footpoint of loop2 and the corresponding intensity evolution (within the yellow box) is presented in panel (i). The black and white contours in panels (a) and (h) represent the negative and positive polarities of the LOS magnetic fields $( \pm 30 \mathrm{G})$ as obtained from HMI.

consistent thermally structured corona, $H_{0}=c_{0} \exp \left(-\frac{y}{\lambda_{0}}\right)$ where $c_{0}=10^{-4} \mathrm{erg} \mathrm{cm}^{-3} \mathrm{~s}^{-1}$ and $\lambda_{0}=80 \mathrm{Mm}$. This heating is used to balance the radiative losses and anisotropic heat conduction related losses of the corona in its equilibrium state. With this initial setup, we integrate the governing MHD equations until the above configuration reaches a quasiequilibrium state, when we reset time to zero.

We use the MPI-parallelized Adaptive Mesh Refinement Versatile Advection Code MPI-AMRVAC (Keppens et al. 2012; Porth et al. 2014) to run the simulation. An effective resolution of $1536 \times 1024$ or an equivalent spatial resolution of $79 \mathrm{~km}$ in both directions is obtained through four AMR levels. The energy release from the flare is mimicked by a finite duration heat pulse $H_{1}$ located at the right footpoint between $x=39,40 \mathrm{Mm}$ :

$$
\begin{gathered}
H_{1}=c_{1} \exp \left(-\left(y-y_{c}\right)^{2} / \lambda^{2}\right) f(t) \\
\text { if } \quad A\left(x_{1}, 0\right)<A(x, y)<A\left(x_{2}, 0\right), \\
A(x, y)=\frac{B_{0} L_{0}}{\pi} \cos \left(\frac{\pi x}{L_{0}}\right) \exp \left(-\frac{\pi y \sin \theta_{0}}{L_{0}}\right),
\end{gathered}
$$

$$
f(t)= \begin{cases}t / 30 & 0 \leqslant t<30 \mathrm{~s} \\ 1 & 30 \leqslant t<150 \mathrm{~s} \\ (180-t) / 30 & 150 \leqslant t<180 \mathrm{~s}\end{cases}
$$

where $\lambda^{2}=10 \mathrm{Mm}^{2}, x_{1}=40 \mathrm{Mm}, y_{c}=3 \mathrm{Mm}$ and $x_{2}=39 \mathrm{Mm}$. The pulse is switched on only for a time $t=0$ to $t=180 \mathrm{~s}$. We set $c_{1}$ to $12 \mathrm{erg} \mathrm{cm}^{-3} \mathrm{~s}^{-1}$ in our simulation input. We introduce the anisotropic thermal conduction along the magnetic field lines with the Spitzer conductivity $\kappa_{\|}$defined as $10^{-6} T^{5 / 2} \mathrm{erg} \mathrm{cm}^{-1} \mathrm{~s}^{-1} \mathrm{~K}^{-3.5}$. We use a radiative loss function of the form $Q=1.2 n_{\mathrm{H}}^{2} \Lambda(T)$ above $10,000 \mathrm{~K}$ (optically thin plasma) (Colgan et al. 2008). Below that value, we set $\Lambda(T)$ to be zero. Density, energy, momentum components ( $y$ and $z$ ), and magnetic field $\left(B_{y}\right.$ and $\left.B_{z}\right)$ are set as symmetric, while $v_{x}$ and $B_{y}$ are taken antisymmetric at the left and right boundaries.

To synthesize the observational features of the SDO/AIA channel we use the FoMo code ${ }^{5}$ to perform forward modeling (Antolin \& Van Doorsselaere 2013; Antolin et al. 2014, 2015;

5 https://wiki.esat.kuleuven.be/FoMo/FrontPage 

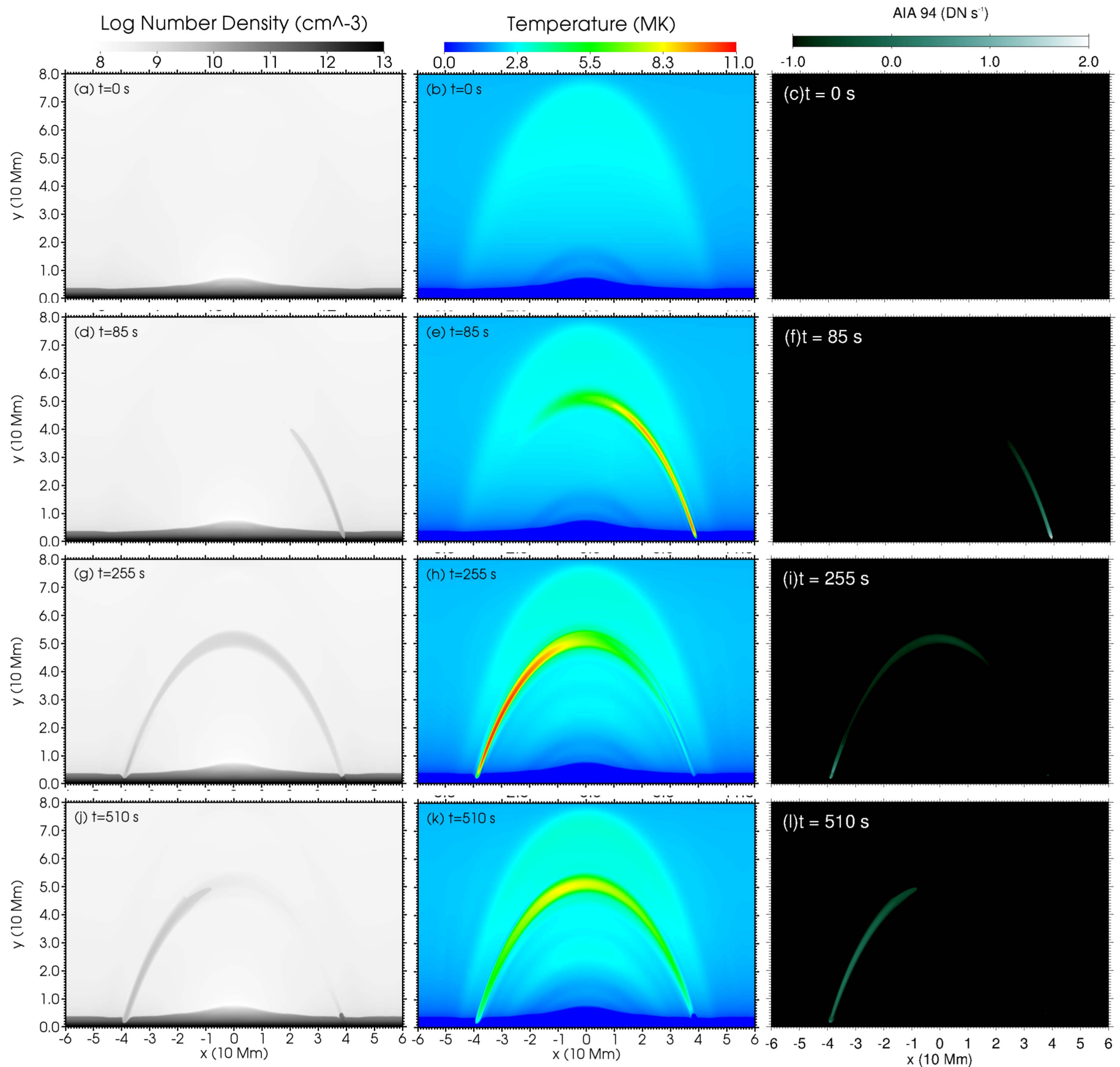

Figure 17. Snapshots showing the density $\left(n_{\mathrm{e}}\right)$, temperature $(T)$ and the AIA $94 \AA$ intensity images respectively at different times of the simulation. The association of the intensity enhancement with the density and temperature is seen very clearly.

(An animation of this figure is available.)

Yuan et al. 2015). Using the AIA temperature response function (Del Zanna et al. 2011; Boerner et al. 2012) the FoMo code converts density to intensity. We synthesized the AIA $94 \AA$ channel emission, which has a characteristic $\log (T) \approx 6.8$.

\subsection{Analysis of the Synthesized Data}

From the movie (movie 7, available online) we see that the wave propagates back and forth before fading out of the loop. Figure 17 shows the time evolution of the density, temperature and the AIA $94 \AA$ channel intensity. To see the wave propagation along the loop we place an artificial slit tracing the loop (red dashed line in the left panel of Figure 18) to generate the time-distance map (right panel of Figure 18). From the map we see clear signatures of reflection in the AIA $94 \AA$ intensity images. The positions of the local maxima were identified along each ridge and fitted with a linear function to calculate the propagation speed.

The two yellow dashed lines represent the fitted straight line for the forward and the reflected wave having speeds $499 \mathrm{~km} \mathrm{~s}^{-1}$ (line A) and $357 \mathrm{~km} \mathrm{~s}^{-1}$ (line B) (with errors less than $15 \mathrm{~km} \mathrm{~s}^{-1}$ ). These speeds compare very well with the average speeds we estimated from time-distance created using the XRT and AIA images. Here we must emphasize that the 

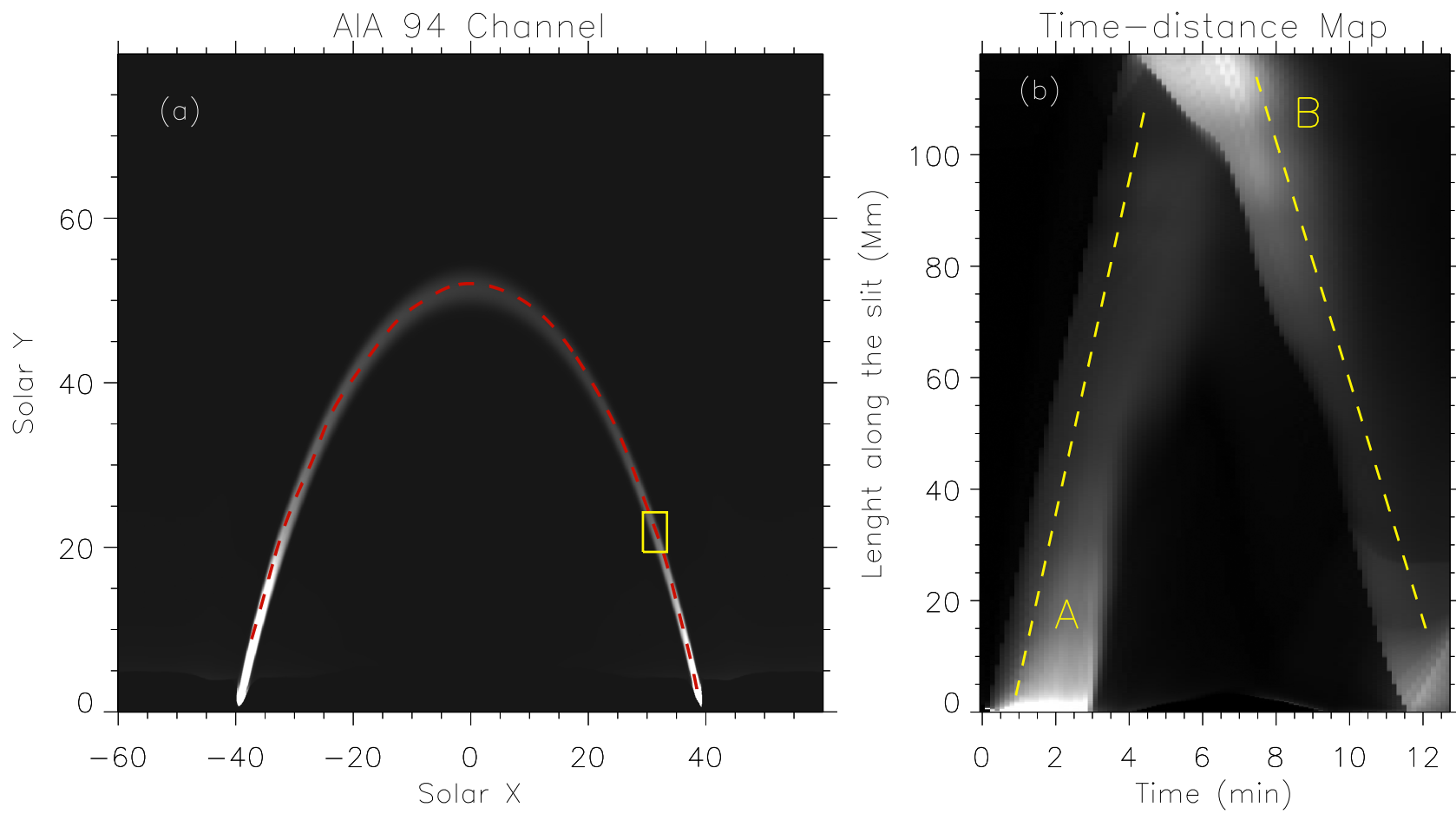

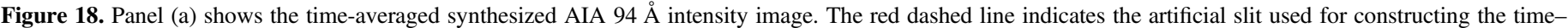

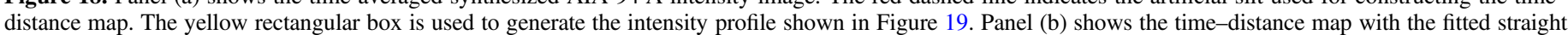
(yellow) line used for speed calculation.

simulation is $2 \mathrm{D}$, so there is no projection effect, unlike our observations. This consistency of the speed value validates our result obtained from DEM analysis which shows a temperature $\approx 10 \mathrm{MK}$.

To estimate the damping of the obsevred propagating intensity, we take a similar approach as for the observational data analysis. The time evolution of the intensity profile averaged over a chosen box (shown as a yellow rectangle in Figure 18) is plotted as a blue solid curve in Figure 19. We fit the function (Equation (1)) on that profile and obtained the best fit curve, shown as the red dotted line. The period and the damping times are 10.1 and $10.3 \mathrm{~min}$ respectively. These values are comparable with the values obtained for the event on December 10.

\section{SUMMARY AND CONCLUSION}

In this paper we report, for the first time, simultaneous observation of a propagating and reflecting intensity disturbance in a hot coronal loop as seen with HINODE/XRT and $S D O / A I A$. We also report three other cases of such reflections of PDs from the XRT. Analysis of the events shows that the observed waves appear after a micro-flare occurs at one of the loop footpoints. The DEM analysis performed on the AIA image sequence reveals the loop temperature and density to be $10 \mathrm{MK}$ and $\approx 10^{9} \mathrm{~cm}^{-3}$ respectively. The average speed of propagation, as estimated from the time-distance maps, is lower than or comparable to the sound speed of the local medium estimated from the DEM analysis. This classifies the wave as a slow propagating mode. We have studied in depth the generation mechanism of such waves and also propose a scenario where a rapidly heated chromospheric plasma acts as a source for the high speed flows we observed in the coronal channels.

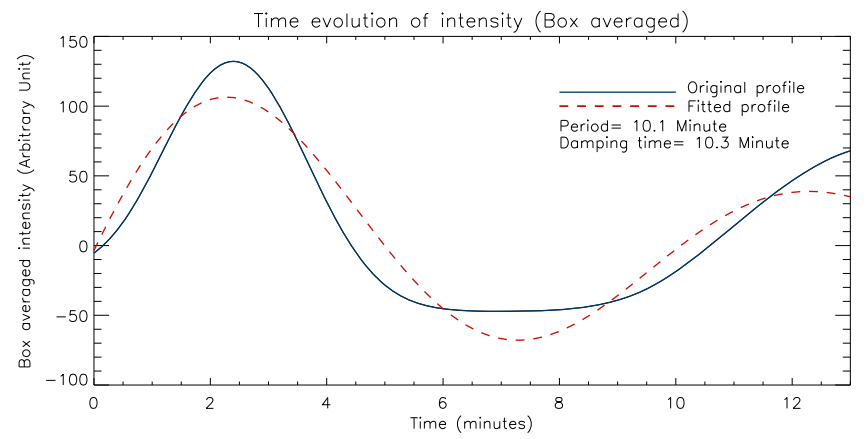

Figure 19. Blue solid line shows the intensity profile averaged over the chosen yellow box. The fitted damped sinusoidal function (Equation (1)) is shown as the red dashed line. The period and the damping time are given on the panel.

From the base-difference images and the time-distance maps we find that the waves are subject to a very fast damping as they propagate along the loop. We have quantitatively measured the damping and found that the damping time $(1 / e$ fall from the initial amplitude) is almost equal to the period of the wave.

We implemented these aspects in a numerical setup where the input loop parameters are the same as obtained from our observations. Injecting a small energy pulse at one of the loop footpoints which mimics the micro-flare trigger, we find a slow MHD wave propagating through the loop and getting reflected back from the other footpoint. We have introduced thermal conduction as the dominant damping factor and found that the wave actually damps very quickly as observed in our events. Analyzing the synthesized data, we have obtained the propagation speeds, which match well with the observed speeds from the XRT and AIA. Using the observed density and temperature values we have reproduced a similar time period of 
the propagating wave from our synthesized AIA data. We also find a good match between the damping time calculated from the synthesized data with the observed damping time. The wave seems to become damped quickly as it propagates through the loop (damping time comparable to the wave period). This fast damping could only be explained by the thermal conduction acting as the major damping mechanism for the propagating slow MHD mode along with the contribution from radiative cooling. Colgan et al. (2008) have estimated the radiation loss from the coronal plasma using different abundance ratio and models and found that the loss due to radiation is $\sim 10^{-22} \mathrm{erg} \mathrm{s}^{-1} \mathrm{~cm}^{3}$. This loss is small compared to the total energy output from the flare which is $\sim 10^{28} \mathrm{erg} \mathrm{s}^{-1}$.

In conclusion we have identified and studied a set of unique events from HINODE/XRT (and SDO/AIA) where a microflare excites a slow MHD wave in a hot coronal loop and the wave is reflected from the other footpoint before fading away. Further study with simultaneous imaging and spectroscopic data, where we can quantify the changes in the time evolution of the line patrameters and their relation to the obsevred brightening, will greatly improve our understanding of the origin as well as the propagation properties of such phenomena.

$S D O$ is a mission for the NASA Living With a Star (LWS) program. Hinode is a Japanese mission developed and launched by ISAS/JAXA, with NAOJ as domestic partner and NASA and STFC (UK) as international partners. It is operated by these agencies in co-operation with ESA and the NSC (Norway). This research has been made possible by the topping-up grant CHARM+top-up COR-SEIS of the BELSPO and the Indian DST. It was also sponsored by an Odysseus grant of the FWO Vlaanderen, and Belspo's IUAP CHARM.

\section{REFERENCES}

Antolin, P., \& Van Doorsselaere, T. 2013, A\&A, 555, A74

Antolin, P., Vissers, G., Pereira, T. M. D., Rouppe van der Voort, L., \& Scullion, E. 2015, ApJ, 806, 81

Antolin, P., Yokoyama, T., \& Van Doorsselaere, T. 2014, ApJL, 787, L22

Aschwanden, M. J., Boerner, P., Schrijver, C. J., \& Malanushenko, A. 2013, SoPh, 283, 5

Aschwanden, M. J., Fletcher, L., Schrijver, C. J., \& Alexander, D. 1999, ApJ, 520,880

Banerjee, D., Erdélyi, R., Oliver, R., \& O’Shea, E. 2007, SoPh, 246, 3

Berghmans, D., \& Clette, F. 1999, SoPh, 186, 207

Boerner, P., Edwards, C., Lemen, J., et al. 2012, SoPh, 275, 41

Colgan, J., Abdallah, J., Jr., Sherrill, M. E., et al. 2008, ApJ, 689, 585

De Moortel, I., \& Hood, A. W. 2003, A\&A, 408, 755

De Moortel, I., \& Hood, A. W. 2004, A\&A, 415, 705

De Moortel, I., Hood, A. W., Gerrard, C. L., \& Brooks, S. J. 2004, A\&A, 425, 741

De Moortel, I., \& Nakariakov, V. M. 2012, RSPTA, 370, 3193
De Pontieu, B., McIntosh, S. W., Hansteen, V. H., \& Schrijver, C. J. 2009, ApJL, 701, L1

DeForest, C. E., \& Gurman, J. B. 1998, ApJL, 501, L217

Del Zanna, G., O’Dwyer, B., \& Mason, H. E. 2011, A\&A, 535, A46

Fang, X., Yuan, D., Van Doorsselaere, T., Keppens, R., \& Xia, C. 2015, ApJ, 813,33

Freeland, S. L., \& Handy, B. N. 1998, SoPh, 182, 497

Golub, L., Deluca, E., Austin, G., et al. 2007, SoPh, 243, 63

Keppens, R., Meliani, Z., van Marle, A. J., et al. 2012, JCoPh, 231, 718

Kiddie, G., De Moortel, I., Del Zanna, G., McIntosh, S. W., \& Whittaker, I. 2012, SoPh, 279, 427

Kosugi, T., Matsuzaki, K., Sakao, T., et al. 2007, SoPh, 243, 3

Kumar, P., Innes, D. E., \& Inhester, B. 2013, ApJL, 779, L7

Kumar, P., Nakariakov, V. M., \& Cho, K.-S. 2015, ApJ, 804, 4

Lemen, J. R., Title, A. M., Akin, D. J., et al. 2012, SoPh, 275, 17

Mandal, S., Samanta, T., Banerjee, D., Krishna Prasad, S., \& Teriaca, L. 2015, RAA, 15, 1832

Mariska, J. T. 2005, ApJL, 620, L67

Mariska, J. T. 2006, ApJ, 639, 484

Mariska, J. T., Warren, H. P., Williams, D. R., \& Watanabe, T. 2008, ApJL, 681, L41

Marsh, M. S., \& Walsh, R. W. 2009, ApJL, 706, L76

Marsh, M. S., Walsh, R. W., \& Plunkett, S. 2009, ApJ, 697, 1674

Moore, R. L., Sterling, A. C., Falconer, D. A., \& Robe, D. 2013, ApJ, 769,134

Nakariakov, V. M., \& Ofman, L. 2001, A\&A, 372, L53

Nakariakov, V. M., Ofman, L., Deluca, E. E., Roberts, B., \& Davila, J. M. 1999, Sci, 285, 862

Nakariakov, V. M., \& Verwichte, E. 2005, LRSP, 2, 3

Ofman, L., Romoli, M., Poletto, G., Noci, G., \& Kohl, J. L. 1997, ApJL, 491, L111

Ofman, L., \& Selwa, M. 2009, in IAU Symp. 257, Three-dimensional MHD modeling of waves in active region loops, ed. N. Gopalswamy \& D. F. Webb (Cambridge: Cambridge Univ. Press), 151

Ofman, L., \& Wang, T. 2002, ApJL, 580, L85

Ofman, L., Wang, T. J., \& Davila, J. M. 2012, ApJ, 754, 111

Pesnell, W. D., Thompson, B. J., \& Chamberlin, P. C. 2012, SoPh, 275, 3

Porth, O., Xia, C., Hendrix, T., Moschou, S. P., \& Keppens, R. 2014, ApJS, 214,4

Roberts, B., Edwin, P. M., \& Benz, A. O. 1984, ApJ, 279, 857

Selwa, M., Murawski, K., \& Solanki, S. K. 2005, A\&A, 436, 701

Selwa, M., \& Ofman, L. 2009, AnGeo, 27, 3899

Selwa, M., Ofman, L., \& Murawski, K. 2007, ApJL, 668, L83

Sterling, A. C., Moore, R. L., Falconer, D. A., \& Adams, M. 2015, Natur, 523,437

Tian, H., McIntosh, S. W., \& De Pontieu, B. 2011, ApJL, 727, L37

Verwichte, E., Marsh, M., Foullon, C., et al. 2010, ApJL, 724, L194

Verwichte, E., Van Doorsselaere, T., White, R. S., \& Antolin, P. 2013, A\&A, 552, A138

Wang, T. 2011, SSRv, 158, 397

Wang, T., Solanki, S. K., Curdt, W., Innes, D. E., \& Dammasch, I. E. 2002, ApJL, 574, L101

Wang, T. J., Solanki, S. K., Curdt, W., et al. 2003a, A\&A, 406, 1105

Wang, T. J., Solanki, S. K., Innes, D. E., \& Curdt, W. 2005, A\&A, 435,753

Wang, T. J., Solanki, S. K., Innes, D. E., Curdt, W., \& Marsch, E. 2003b, A\&A, 402, L17

Yuan, D., Van Doorsselaere, T., Banerjee, D., \& Antolin, P. 2015, ApJ, 807, 98 